MPP-2007-54

\title{
Collective neutrino flavor transitions in supernovae and the role of trajectory averaging
}

\author{
Gianluigi Fogli ${ }^{1,2}$, Eligio Lisi ${ }^{2}$, Antonio Marrone ${ }^{1,2}$, Alessandro \\ Mirizzi $^{1,2,3}$ \\ ${ }^{1}$ Dipartimento Interateneo di Fisica "Michelangelo Merlin", Via Orabona 4, 70126 \\ Bari, Italy \\ ${ }^{2}$ Istituto Nazionale di Fisica Nucleare, Sezione di Bari, Via Orabona 4, 70126 Bari, \\ Italy \\ ${ }^{3}$ Max-Planck-Institut für Physik (Werner-Heisenberg-Institut), Föhringer Ring 6, \\ 80805 München, Germany
}

\begin{abstract}
Non-linear effects on supernova neutrino oscillations, associated with neutrino-neutrino interactions, are known to induce collective flavor transformations near the supernova core for $\theta_{13} \neq 0$. In scenarios with very shallow electron density profiles, these transformations have been shown to couple with ordinary matter effects, jointly producing spectral distortions both in normal and inverted hierarchy. In this work we consider a complementary scenario, characterized by higher electron density, as indicated by shock-wave simulations during a few seconds after bounce. In this case, early collective flavor transitions are decoupled from later, ordinary matter effects. Moreover, such transitions become more amenable to both numerical computations and analytical interpretations in inverted hierarchy, while they basically vanish in normal hierarchy. We numerically evolve the neutrino density matrix in the region relevant for self-interaction effects, using thermal spectra and a representative value $\sin ^{2} \theta_{13}=10^{-4}$. In the approximation of averaged intersection angle between neutrino trajectories, our simulations neatly show the collective phenomena of synchronization, bipolar oscillations, and spectral split, with analytically understandable features, as recently discussed in the literature. In the more realistic (but computationally demanding) case of non-averaged neutrino trajectories, our simulations do not show new significant qualitative features, apart from the smearing of "fine structures" such as bipolar nutations. Our results seem to suggest that, at least for non-shallow matter density profiles, averaging over neutrino trajectories plays a minor role in the final outcome. In this case, the swap of $\nu_{e}$ and $\nu_{\mu, \tau}$ spectra above a critical energy may represent an unmistakable signature of the inverted neutrino hierarchy, especially for $\theta_{13}$ small enough to render further (ordinary or even turbulent) matter effects irrelevant.
\end{abstract}

PACS numbers: 14.60.Pq, 13.15.+g, 97.60.Bw 
Collective neutrino flavor transitions in supernovae and the role of trajectory averaging 2

\section{Introduction}

Core-collapse supernovae (SN) provide us with an interesting laboratory for studying both neutrino properties and their interplay with astrophysical processes (see $[1,2]$ for recent reviews). Mikheev-Smirnov-Wolfenstein (MSW) effects induced on neutrinos by background matter [3] have been widely studied as a tool to probe both neutrino masses and mixings and the SN dynamics. Recent examples include the characterization, at the level of observable SN neutrino event spectra, of shock-wave evolution effects after bounce [4-13] and of it possible "erasing" by stochastic matter fluctuations induced by turbulence [14-16].

Ordinary MSW effects (and their "stochastic" smearing, if any) typically occur when $\omega \sim \lambda$, where

$$
\omega=\frac{\Delta m^{2}}{2 E}
$$

is the vacuum oscillation frequency (in terms of the neutrino energy $E$ and squared mass difference $\left.\Delta m^{2}\right) \ddagger$ while

$$
\lambda(r)=\sqrt{2} G_{F} N_{e^{-}}(r)
$$

is the $\nu_{e^{-}} \nu_{x}$ interaction energy difference in matter, $N_{e^{-}}(r)$ being the net electron number density at the point $r$. For typical shock-wave density profiles as used, e.g., in $[7,8]$, the condition $\omega \sim \lambda$ occurs after a few hundred (or even a few thousand) kilometers, during the first few seconds after core bounce. Significantly shallower electron density profiles (as those adopted in [17] in the context of models with successful $r$-process nucleosynthesis) can instead trigger MSW effects much earlier, around $O(100) \mathrm{km}$.

Besides electrons, neutrinos can also be a nontrivial background to themselves when their density is large enough $[18,19]$. Self-interaction effects, being inherently non-linear, are very different (and much less intuitive) than ordinary MSW effect, and can lead to collective flavor transition phenomena in which neutrinos (or antineutrinos) of any energy behave similarly [20-34]. The interest of such effects for the neutrino flavor evolution in the dense SN core has long been recognized [35-39]. Roughly speaking, significant self-interaction phenomena are expected when $\mu(r) \gtrsim \omega$, where

$$
\mu(r)=\sqrt{2} G_{F}[N(r)+\bar{N}(r)]
$$

$N(r)$ and $\bar{N}(r)$ being the total effective neutrino $\left(\nu_{e}+\nu_{x}\right)$ and antineutrino $\left(\bar{\nu}_{e}+\bar{\nu}_{x}\right)$ number density, respectively (to be precisely defined later).

In the most general case, systems with dense matter and dense neutrino gases are thus governed by (at least) three characteristic frequencies: $\omega$ (spread over $\sim 2$ orders of magnitude for typical energy spectra); $\lambda$ (roughly decreasing as the third power of the distance); and $\mu$ (decreasing as the fourth power of the distance [17]). Neutrino flavor evolution becomes then a complicated multi-scale dynamical problem with a rich

$\ddagger$ In this paper we neglect $\delta m^{2}=m_{2}^{2}-m_{1}^{2} \ll \Delta m^{2}$, where $\Delta m^{2}=\left|m_{3}^{2}-m_{1,2}^{2}\right|$. The only relevant mixing angle is then $\theta_{13}$, governing the oscillation amplitude in the channels $\nu_{e} \rightarrow \nu_{x}$ and $\bar{\nu}_{e} \rightarrow \bar{\nu}_{x}$ $(x=\mu$ or $\tau)$. 
phenomenology, which may involve both collective and MSW effects, the latter being often assumed (at least in older literature) to lead to flavor transitions. In the SN neutrino context, this "old" paradigm (focussing on MSW effects) has dramatically changed after the emergence of dominant collective phenomena (such as the so-called "bipolar oscillations") studied in detailed, large-scale computer simulations [17,40] as well as in simplified but analytical models [41-47].

The interaction strength between two (anti)neutrinos is modulated by a factor $\left(1-\cos \vartheta_{i j}\right)$, where $\vartheta_{i j}$ is the angle between their intersecting trajectories. If one ignores the spread of $\vartheta_{i j}$, and averages it out along a "representative" radial trajectory (single-angle approximation), and if one also assumes that self-interaction effects do not interfere with the ordinary MSW ones, then the following picture appears to emerge in supernovae from analytical considerations. Nothing relevant occurs for normal hierarchy $\left(m_{3}>m_{1,2}\right)$, while, for inverted mass hierarchy $\left(m_{3}<m_{1,2}\right)$, any value of $\theta_{13} \neq 0$ (no matter how small $[42,43]$ ) can trigger collective pair-conversions of the kind $\nu_{e} \bar{\nu}_{e} \rightarrow \nu_{x} \bar{\nu}_{x}$ within the first $O(100) \mathrm{km}$. Then, as recently emphasized in $[45,46]$, the end of collective effects is marked by a spectral pair-conversion which is complete for $\bar{\nu}$ 's, while for $\nu$ 's it occurs only above a characteristic energy set by lepton number conservation. Such spectral "split" (or "stepwise swap" of flavors) would then represent an unmistakable signature of self-interaction effects [17, 40,44-48]. Its robustness needs, however, to be better investigated in increasingly refined SN models including, e.g., variable neutrino crossing angles $\vartheta_{i j}$ (multi-angle simulations), which might induce kinematical decoherence effects, ("depolarization" and "smearing of oscillations"), which are neither obvious nor entirely clear in the few numerical $[17,49]$ and analytical $[34,43]$ studies performed so far. In our scenario, it turns out that main results are rather robust when passing from single- to multi-angle simulations.

The purpose of this paper is to explore such "self-interaction dominated" scenario in a realistic case characterized by: (1) an appropriate matter profile, where collective effects fully develop before MSW effects (if any); (2) continuous, thermal energy spectra with significant neutrino-antineutrino (and neutrino flavor) asymmetry, and (3) numerical simulations in single- and multi-angle cases. In this sense, our work is complementary to the simulations in [17], where the shallower adopted matter profile allows MSW effects to occur within (not beyond) the range of collective transitionswhich leads to a richer phenomenology, but also to more difficult analyses and much greater numerical challenges. In the terminology of [42], we study the scenario with "thick" rather than "thin" envelope.

The plan of our work is as follows. In Section 2 we describe our supernova reference model. In Section 3 we set the notation and write the neutrino evolution equations in single-angle approximation. In Section 4 and 5 we discuss single-angle analytical and numerical solutions, respectively. In Section 5 we tackle multi-angle simulations, and show that the main final effect (the spectral split) is robust. Conclusions and perspectives are presented in Section 6. Technical aspects are discussed in the Appendix. 
Collective neutrino flavor transitions in supernovae and the role of trajectory averaging 4

\section{Reference Supernova Model}

Our reference model is characterized by the following choices for the initial neutrino energy spectra, the geometry and intensity of neutrino emission, and the matter profile, which govern the distribution of the three basic "frequencies" $\omega, \mu$, and $\lambda$, respectively.

We shall use such choices in the usual, simplified context of pure two-family $\left(\nu_{e}, \nu_{x}\right)$ evolution $[42,43]$, where $\nu_{x}$ represents a single active flavor. It is worth noticing that, for $\lambda \neq 0$, this case does not exactly represent the two-family limit (for $\delta m^{2}=0$ ) of the general three-family case, contrary to the familiar MSW effects in SNe. In fact, in the presence of self-interactions, the flavor evolution depends on the absolute effective neutrino densities and, thus, also on the total number of neutrino families (either two or three) assumed to share the total luminosity. The full $3 \nu$ case (and its proper $2 \nu$ limit) will be studied elsewhere.

\subsection{Initial Neutrino Energy Spectra}

We assume normalized thermal spectra with different temperatures $T=1 / \beta$ for $\nu_{e}, \bar{\nu}_{e}$, and $\nu_{x}$ (the latter having the same properties as $\bar{\nu}_{x}$ ). More precisely, the initial energy spectra $\phi^{i}(E)$ are of the form

$$
\phi^{i}(E)=\frac{2 \beta}{3 \zeta_{3}} \frac{(\beta E)^{2}}{e^{\beta E}+1},
$$

where $\zeta_{3} \simeq 1.202$. The average values of $E$ and $E^{-1}$ are then:

$$
\left\langle E^{ \pm 1}\right\rangle=\int d E \phi(E) E^{ \pm 1}=c_{ \pm} T^{ \pm 1}=c_{ \pm} \beta^{\mp 1}
$$

where $c_{+}=7 \pi^{4} / 180 \zeta_{3} \simeq 3.151$ and $c_{-}=\pi^{2} / 18 \zeta_{3} \simeq 0.4561$. In numerical calculations we choose $\left\langle E_{e}\right\rangle=10 \mathrm{MeV},\left\langle\bar{E}_{e}\right\rangle=15 \mathrm{MeV}$, and $\left\langle E_{x}\right\rangle=\left\langle\bar{E}_{x}\right\rangle=24 \mathrm{MeV}$ for $\nu_{e}, \bar{\nu}_{e}, \nu_{x}$ and $\bar{\nu}_{x}$, respectively, corresponding to

$$
\beta_{e}=0.315, \bar{\beta}_{e}=0.210, \beta_{x}=\bar{\beta}_{x}=0.131\left(\mathrm{MeV}^{-1}\right) .
$$

\subsection{Emission Geometry and Intensity}

We adopt the "bulb model" emission described in [17], to which the reader is referred for further details. Neutrinos are assumed to be half-isotropically emitted above the neutrino-sphere, i.e., all the outward moving modes are equally occupied as expected from a blackbody emission. The neutrino number flux $j_{\nu}$ per unit energy (in any direction) is then given by [17]

$$
j_{\nu}(E)=\frac{F_{\nu}}{2 \pi} \frac{\phi^{i}(E)}{\langle E\rangle},
$$

where

$$
F_{\nu}=\frac{L_{\nu}}{2 \pi R_{\nu}^{2}}
$$


Collective neutrino flavor transitions in supernovae and the role of trajectory averaging 5

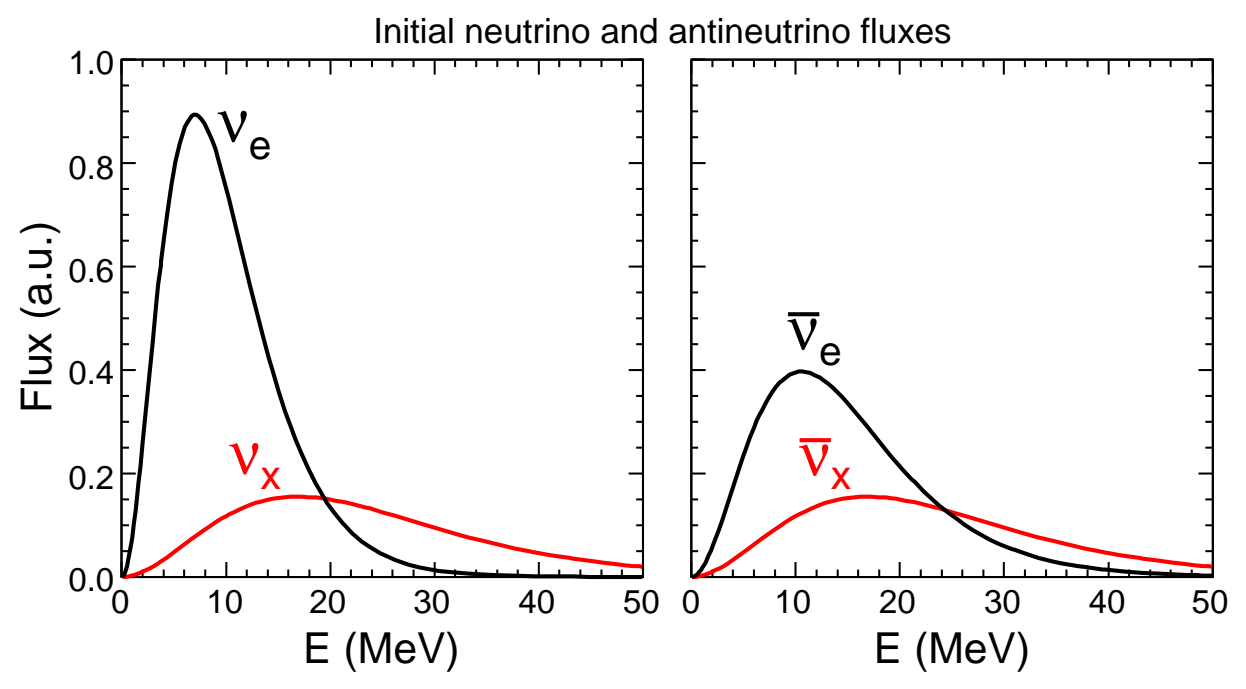

Figure 1. Initial fluxes (at $r=10 \mathrm{~km}$, in arbitrary units) for different neutrino species as a function of energy. The fluxes are all proportional to $\phi^{i}(E) /\langle E\rangle$.

$R_{\nu}$ being the neutrino-sphere radius, while $L_{\nu}$ is the total emission power for a given neutrino species. In numerical calculations, we assume reference values $R_{\nu}=10 \mathrm{~km}$ and $L_{\nu}=10^{51} \mathrm{erg} / \mathrm{s}$ for each species $\nu=\nu_{e}, \bar{\nu}_{e}, \nu_{x}, \bar{\nu}_{x}$.

Figure 1 shows the initial neutrino number fluxes per unit energy in arbitrary units (all fluxes being proportional to $\phi^{i}(E) /\langle E\rangle$ through the same normalization constant). Notice the significant difference (asymmetry) between neutrinos and antineutrinos, and between different neutrino flavors. However, the $\nu_{e}$ and $\nu_{x}$ fluxes happen to coincide at an energy $E_{\text {eq }} \simeq 19 \mathrm{MeV}$, while for the $\bar{\nu}_{e}$ and $\bar{\nu}_{x}$ fluxes the equality occurs at $\bar{E}_{\text {eq }} \simeq 24 \mathrm{MeV}$. Flavor transformations of any kind are not operative for neutrinos at $E=E_{\text {eq }}$, and for antineutrinos at $E=\bar{E}_{\text {eq }}$.

The spherical symmetry of emission reduces to a cylindrical symmetry along the radial line-of-sight (polar axis). At any radius $r>R_{\nu}$ along the polar axis, neutrinos will arrive with different momenta $\mathbf{p}$ characterized by $|\mathbf{p}|=E$, incident polar angle $\vartheta$, and azimuthal angle $\varphi$. In the calculation of self-interaction effects, the effective differential neutrino number density $d n_{\mathbf{p}}$ with momentum between $\mathbf{p}$ and $\mathbf{p}+d \mathbf{p}$ is then [17]

$$
d n_{\mathbf{p}}=j_{\nu}(E) d \Omega=j_{\nu}(E) d \varphi d \cos \vartheta,
$$

within the cone of sight of the neutrino-sphere, with $\vartheta \in\left[0, \vartheta_{\max }\right]$, being

$$
\vartheta_{\max }=\arcsin \left(R_{\nu} / r\right) \text {. }
$$

In general, angular coordinates are important, since the interaction strength between two neutrinos of momenta $\mathbf{p}$ and $\mathbf{q}$ depends on their relative angle $\vartheta_{\mathbf{p q}}$ through the factor $\left(1-\cos \vartheta_{\mathbf{p q}}\right)$. Calculations embedding the full angular coordinates are dubbed "multi-angle." The often used "single-angle" approximation consists in averaging the angular factor along the polar axis, which is assumed to encode the same flavor history of any other neutrino direction. In this case, the effective neutrino number density $n$ 
Collective neutrino flavor transitions in supernovae and the role of trajectory averaging 6

per unit volume and energy turns out to be [17]

$$
n(r, E)=2 \pi D(r) j_{\nu}(E)=F_{\nu} D(r) \frac{\phi^{i}(E)}{\langle E\rangle}
$$

for the various neutrino species $\left(n=n_{e}, \bar{n}_{e}, n_{x}\right.$ or $\left.\bar{n}_{x}\right)$, where the (species-independent) geometrical function $D(r)$ is given by

$$
D(r)=\frac{1}{2}\left[1-{\sqrt{1-\left(\frac{R_{\nu}}{r}\right)^{2}}}^{2},\right.
$$

decreasing as $r^{-4}$ for large $r$. The $\nu$ - $\bar{\nu}$ asymmetry of the model implies $n_{e} \neq \bar{n}_{e}$.

It is useful to integrate the effective densities per unit energy and volume $\left(n_{e}, \bar{n}_{e}\right.$, and $\left.n_{x}=\bar{n}_{x}\right)$ to obtain the effective number densities of $\nu_{e}, \bar{\nu}_{e}$ and $\nu_{x}\left(\bar{\nu}_{x}\right)$,

$$
\begin{aligned}
& N_{e}=\int d E n_{e}=F_{\nu} \frac{D(r)}{\left\langle E_{e}\right\rangle}=\frac{F_{\nu}}{c_{+}} D(r) \beta_{e}, \\
& \bar{N}_{e}=\int d E \bar{n}_{e}=F_{\nu} \frac{D(r)}{\left\langle\bar{E}_{e}\right\rangle}=\frac{F_{\nu}}{c_{+}} D(r) \bar{\beta}_{e}, \\
& N_{x}=\int d E n_{x}=F_{\nu} \frac{D(r)}{\left\langle E_{x}\right\rangle}=\frac{F_{\nu}}{c_{+}} D(r) \beta_{x}=\bar{N}_{x}
\end{aligned}
$$

as well as the total effective number densities of neutrinos and antineutrinos,

$$
\begin{aligned}
& N=N_{e}+N_{x}, \\
& \bar{N}=\bar{N}_{e}+\bar{N}_{x},
\end{aligned}
$$

from which one can finally derive the parameter $\mu=\sqrt{2} G_{F}(N+\bar{N})$, which governs the neutrino self-interaction strength.

Figure 2 shows the function $\mu(r)$ in our reference SN model (together with the ordinary MSW strength $\lambda(r)$ defined below) in the range $r \in[10,200] \mathrm{km}$ relevant for self-interaction effects. Also shown are the approximate ranges where these effects induce synchronization, bipolar oscillations and spectral split, as discussed later in Sec. 3 .

\subsection{Matter Profile}

Shock waves propagating during the first few second after bounce can produce very interesting MSW effects when $\lambda \sim \omega$, provided that $\sin ^{2} \theta_{13}$ is not too small [4-11]. For the typical (time-dependent) matter profiles used in these studies, such effects usually emerge in the first $\sim 10$ s after a few hundred kilometers from the SN core, in which case collective neutrino self-interaction effects are already completely developed, as we shall see later. In this case, the specific choice of the neutrino potential profile $\lambda(r)=\sqrt{2} G_{f} N_{e^{-}}$turns out to have only a minor impact on the evolution of selfinteraction effects, even if $\lambda(r) \gg \mu(r)$. For the sake of definiteness, we single out one of the time-dependent profiles studied in [7] (the one at post-bounce time $t=5 \mathrm{~s}$ ), and steepen it in a few $\mathrm{km}$ range above the neutrino sphere as suggested in $[17,50] . \S$ The resulting profile $\lambda(r)$ adopted in this work is shown in Figure 2.

$\S$ The local steepening does not change any feature of the collective neutrino flavor transformations, but it turns out to help the start-up of our numerical evolution routines in the first few $\mathrm{km}$. 


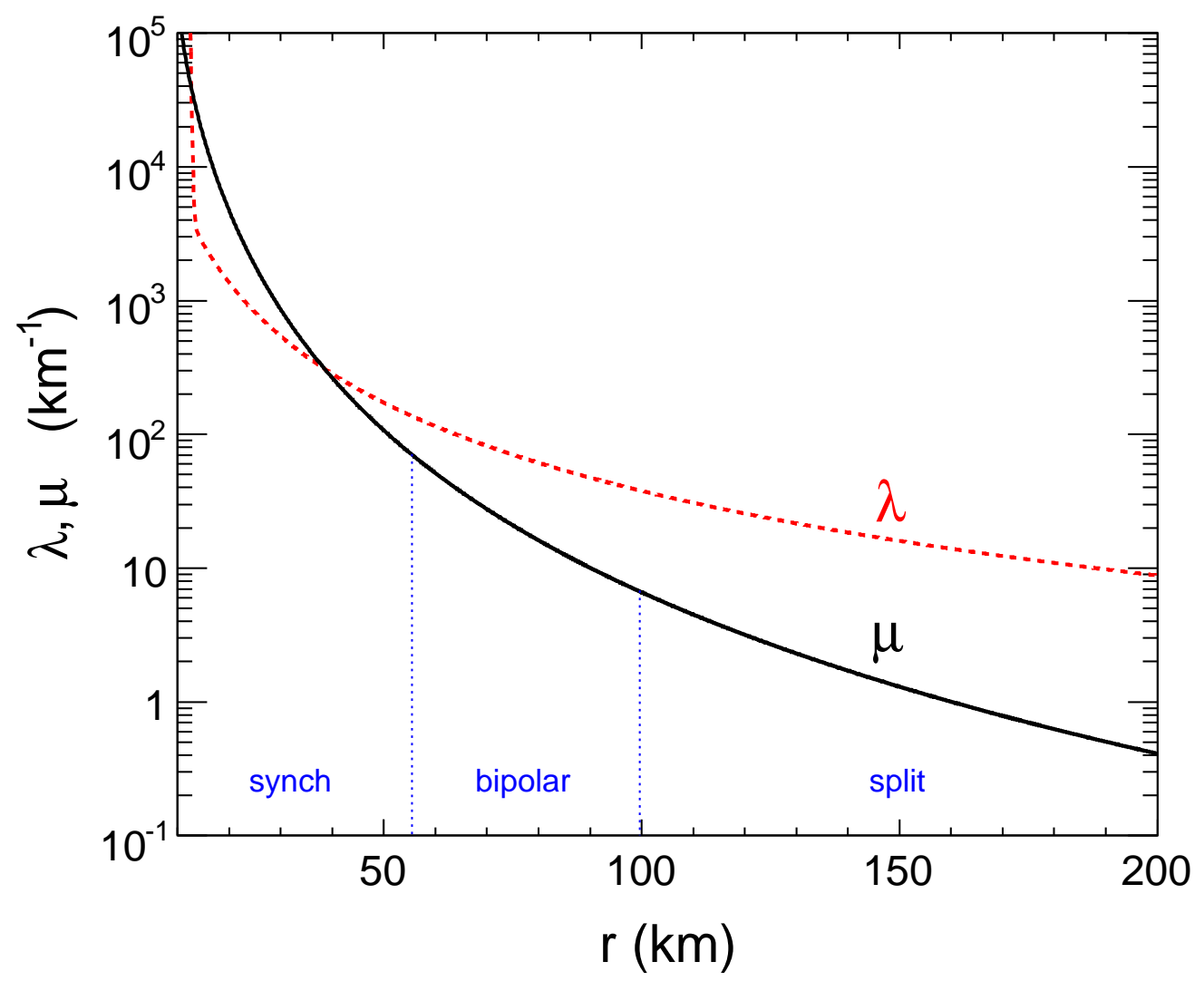

Figure 2. Radial profiles of the neutrino self-interaction parameter $\mu(r)=$ $\sqrt{2} G_{F}(N+\bar{N})$ and of the matter-interaction parameter $\lambda(r)=\sqrt{2} G_{F} N_{e^{-}}$adopted in this work, in the range $r \in[10,200] \mathrm{km}$. Shown are also the approximative ranges where self-interaction effects are expected to produce mainly synchronization, bipolar oscillations and spectral split (see the text for details).

\section{Single-Angle Approximation: Analytical aspects}

An ensemble of relativistic neutrinos and antineutrinos coming in $F$ flavors can be described by a set of dimensionless $F \times F$ density matrices $\rho_{\mathbf{p}}$ and $\bar{\rho}_{\mathbf{p}}$, one for each momentum mode. The most general Liouville evolution equations for $\rho_{\mathbf{p}}$ have been worked out in [51]. In this Section we specialize and discuss such equations in Bloch form for $F=2$ and single-angle approximation. Although we mostly rely on the formalism introduced in [43] and on the results presented in [17,42-45], we think it useful-for the sake of clarity and self-consistency - to give a systematic overview of the asymmetric $\left(n_{e} \neq \bar{n}_{e}\right)$ and continuous-energy case, also because our choice of thermal spectra allows some useful analytical estimates in terms of temperature parameters $\beta=1 / T$. 
Collective neutrino flavor transitions in supernovae and the role of trajectory averaging 8

\subsection{Bloch Vector Notation}

We consider two-family mixing between $\nu_{e}$ and $\nu_{x}\left(\bar{\nu}_{e}\right.$ and $\left.\bar{\nu}_{x}\right)$, driven by mass-mixing parameters $\left(\Delta m^{2}, \sin ^{2} \theta_{13}\right)$. In this case, one can switch from normal to inverted mass hierarchy in two ways: (1) mixing is kept unaltered while $+\Delta m^{2} \rightarrow-\Delta m^{2}$; or (2) $\Delta m^{2}$ is kept positive, while the mixing angle is swapped, $\sin \theta_{13} \leftrightarrow \cos \theta_{13}$ (which implies $+\cos 2 \theta_{13} \rightarrow-\cos 2 \theta_{13}$, with unaltered $\left.\sin 2 \theta_{13}\right)$. Hereafter we adopt the latter choice, as advocated in [43]. In numerical calculations, we assume reference values

$$
\Delta m^{2}=2 \times 10^{-3} \mathrm{eV}^{2}, \quad \sin ^{2} \theta_{13}=10^{-4} .
$$

In the single-angle approximation, the only kinematical parameter is $E=|\mathbf{p}|$. For each energy $E$ and radius $r$, the neutrino density matrix $\rho$ in flavor basis can be written in terms of polarization (Bloch) vector $\mathbf{P}=\left(P_{x}, P_{y}, P_{z}\right)^{T}=P_{x} \mathbf{x}+P_{y} \mathbf{y}+P_{z} \mathbf{z}$, being $\mathbf{x}, \mathbf{y}$ and $\mathbf{z}$ three orthogonal unit vectors, and of the vector of Pauli matrices $\boldsymbol{\sigma}=\left(\sigma_{x}, \sigma_{y}, \sigma_{z}\right)^{T}$

$$
\rho=\left(\begin{array}{cc}
\rho_{e e} & \rho_{e x} \\
\rho_{x e} & \rho_{x x}
\end{array}\right)=\left(\begin{array}{ll}
\left|\nu_{e}\right|^{2} & \nu_{e} \nu_{x}^{*} \\
\nu_{e}^{*} \nu_{x} & \left|\nu_{x}\right|^{2}
\end{array}\right)=\frac{n}{2}(\mathbf{1}+\mathbf{P} \cdot \boldsymbol{\sigma}),
$$

where 1 is the unit matrix, $n=\operatorname{Tr}(\rho)=n_{e}+n_{x}$ represents the occupation number of neutrinos (per unit volume) at energy $E$, and the densities are defined through Eq. (11) in our model. Analogous definitions in terms of $\overline{\mathbf{P}}$ hold for antineutrinos. The initial conditions in flavor basis $\rho^{i}=\operatorname{diag}\left(n_{e}, n_{x}\right)$ and $\bar{\rho}^{i}=\operatorname{diag}\left(\bar{n}_{e}, \bar{n}_{x}\right)$ correspond to

$$
\begin{aligned}
& \mathbf{P}^{i}=P_{z}^{i} \mathbf{z}=\frac{n_{e}-n_{x}}{n} \mathbf{z}, \\
& \overline{\mathbf{P}}^{i}=\bar{P}_{z}^{i} \mathbf{z}=\frac{\bar{n}_{e}-\bar{n}_{x}}{\bar{n}} \mathbf{z} .
\end{aligned}
$$

The final $(f)$ survival probabilities are then given by

$$
\begin{aligned}
& P_{e e}=P\left(\nu_{e}^{i} \rightarrow \nu_{e}^{f}\right)=\frac{1}{2}\left(1+\frac{P_{z}^{f}}{P_{z}^{i}}\right), \\
& \bar{P}_{e e}=P\left(\bar{\nu}_{e}^{i} \rightarrow \bar{\nu}_{e}^{f}\right)=\frac{1}{2}\left(1+\frac{\bar{P}_{z}^{f}}{\bar{P}_{z}^{i}}\right) .
\end{aligned}
$$

It is useful to introduce the integral polarization vectors of neutrinos and antineutrinos,

$$
\begin{aligned}
& \mathbf{J}=\frac{1}{N+\bar{N}} \int d E n \mathbf{P}, \\
& \overline{\mathbf{J}}=\frac{1}{N+\bar{N}} \int d E \bar{n} \overline{\mathbf{P}},
\end{aligned}
$$

as well as their sum $\mathbf{S}$ and difference $\mathbf{D}$,

$$
\begin{aligned}
& \mathbf{S}=\mathbf{J}+\overline{\mathbf{J}}, \\
& \mathbf{D}=\mathbf{J}-\overline{\mathbf{J}} .
\end{aligned}
$$


Collective neutrino flavor transitions in supernovae and the role of trajectory averaging 9

The initial conditions imply that

$$
\begin{aligned}
\mathbf{J}^{i} & =\frac{N_{e}-N_{x}}{N+\bar{N}} \mathbf{z}=\frac{\beta_{e}-\beta_{x}}{\beta_{e}+\bar{\beta}_{e}+2 \beta_{x}} \mathbf{z}, \\
\overline{\mathbf{J}}^{i} & =\frac{\bar{N}_{e}-\bar{N}_{x}}{N+\bar{N}} \mathbf{z}=\frac{\bar{\beta}_{e}-\beta_{x}}{\beta_{e}+\bar{\beta}_{e}+2 \beta_{x}} \mathbf{z}, \\
\mathbf{S}^{i} & =\frac{N_{e}+\bar{N}_{e}-2 N_{x}}{N+\bar{N}} \mathbf{z}=\frac{\beta_{e}+\bar{\beta}_{e}-2 \beta_{x}}{\beta_{e}+\bar{\beta}_{e}+2 \beta_{x}} \mathbf{z}, \\
\mathbf{D}^{i} & =\frac{N_{e}-\bar{N}_{e}}{N+\bar{N}} \mathbf{z}=\frac{\beta_{e}-\bar{\beta}_{e}}{\beta_{e}+\bar{\beta}_{e}+2 \beta_{x}} \mathbf{z} .
\end{aligned}
$$

Another auxiliary (unit) vector is the "magnetic field,"

$$
\mathbf{B}=\sin 2 \theta_{13} \mathbf{x} \mp \cos 2 \theta_{13} \mathbf{z}
$$

where the upper (lower) sign refers to normal (inverted) hierarchy. For small $\theta_{13}$, one can take $\mathbf{B} \simeq \mp \mathbf{z}$, unless the relevant dynamics is very close to the $z$ axis.

\subsection{Equations Of Motion And Removal of Matter Effects}

The Bloch equations of motion (EOM) for each polarization vector $\mathbf{P}$ read, in singleangle approximation,

$$
\begin{aligned}
& \dot{\mathbf{P}}=(+\omega \mathbf{B}+\lambda \mathbf{z}+\mu \mathbf{D}) \times \mathbf{P}, \\
& \dot{\overline{\mathbf{P}}}=(-\omega \mathbf{B}+\lambda \mathbf{z}+\mu \mathbf{D}) \times \overline{\mathbf{P}},
\end{aligned}
$$

where the three terms in brackets embed vacuum, matter, and self-interaction effects. In particular, the third term couples all modes $\mathbf{P}$ and $\mathbf{P}$, and is responsible for collective effects. The equations conserve each $|\mathbf{P}|$ and thus unitarity. It is understood that $\mathbf{P}=\mathbf{P}(E, r), \lambda=\lambda(r)$, and $\mu=\mu(r)$, with $r=t$ and $d_{t}=d_{r}$. If the continuous parameter $E$ is discretized through a set of $N_{E}$ points $\left\{E_{h}\right\}_{h=1, \ldots, N_{E}}$, then a set of $6 \times N_{E}$ coupled, first-order differential equations in $t$ is obtained. The equations are "stiff," namely, their solutions generally involve a fast-changing combination of multifrequency oscillations, due to the "precession" of $\mathbf{P}$ and $\overline{\mathbf{P}}$ around the three terms in brackets. It is thus amazing that, through appropriate approximations, the resulting dynamics turns out to be understandable in terms of simple phenomena, as discussed below.

In a frame rotating with angular velocity $\lambda \mathbf{z}[42]$, the time derivative acquires an extra operator $-\lambda \mathbf{z} \times$, which cancels the matter term. Moreover, the $z$-component of $\mathbf{P}$ (and of any other vector) are unchanged in such co-rotating frame, and thus the survival probability $P_{e e}$ is also unchanged. Only the transverse $(x, y)$ components of any vector get mixed, e.g., the "magnetic field" becomes $\mathbf{B}=$ $\left(\sin 2 \theta_{13} \cos (-\lambda t), \sin 2 \theta_{13} \sin (-\lambda t), \mp \cos 2 \theta_{13}\right)^{T}$. Again, one can still take $\mathbf{B} \simeq \mp \mathbf{z}$, unless the relevant dynamics does not occur too close to $\mathbf{B}$ or $\mathbf{z}$ (a case which will be discussed separately). Matter $(\lambda)$ effects thus "disappear" in such co-rotating frame, as 
Collective neutrino flavor transitions in supernovae and the role of trajectory averaging 10

pointed out in [42], leaving the other terms in the EOM formally unchanged:

$$
\begin{aligned}
& \dot{\mathbf{P}}=(+\omega \mathbf{B}+\mu \mathbf{D}) \times \mathbf{P}, \\
& \dot{\bar{P}}=(-\omega \mathbf{B}+\mu \mathbf{D}) \times \overline{\mathbf{P}} .
\end{aligned}
$$

The corresponding EOM for integral quantities are:

$$
\begin{aligned}
\dot{\mathbf{J}} & =+\mathbf{B} \times \mathbf{W}+\mu \mathbf{D} \times \mathbf{J}, \\
\dot{\mathbf{J}} & =-\mathbf{B} \times \overline{\mathbf{W}}+\mu \mathbf{D} \times \overline{\mathbf{J}}, \\
\dot{\mathbf{S}} & =\mathbf{B} \times(\mathbf{W}-\overline{\mathbf{W}})+\mu \mathbf{D} \times \mathbf{S}, \\
\dot{\mathbf{D}} & =\mathbf{B} \times(\mathbf{W}+\overline{\mathbf{W}}),
\end{aligned}
$$

where

$$
\begin{aligned}
& \mathbf{W}=\frac{1}{N+\bar{N}} \int d E \omega n \mathbf{P}, \\
& \overline{\mathbf{W}}=\frac{1}{N+\bar{N}} \int d E \omega \bar{n} \overline{\mathbf{P}},
\end{aligned}
$$

with initial conditions implying

$$
\begin{aligned}
\mathbf{W}^{i} & =\frac{1}{N+\bar{N}} \int d E \omega\left(n_{e}-n_{x}\right) \mathbf{z}=\frac{\Delta m^{2} c_{-}}{2} \frac{\beta_{e}^{2}-\beta_{x}^{2}}{\beta_{e}+\bar{\beta}_{e}+2 \beta_{x}} \mathbf{z}, \\
\overline{\mathbf{W}}^{i} & =\frac{1}{N+\bar{N}} \int d E \omega\left(\bar{n}_{e}-\bar{n}_{x}\right) \mathbf{z}=\frac{\Delta m^{2} c_{-}}{2} \frac{\bar{\beta}_{e}^{2}-\beta_{x}^{2}}{\beta_{e}+\bar{\beta}_{e}+2 \beta_{x}} \mathbf{z} .
\end{aligned}
$$

\subsection{Conservation Laws and Spectral Split}

The equation for $\dot{\mathbf{D}}$ implies conservation of the scalar

$$
\mathbf{D} \cdot \mathbf{B}=\text { const }=\mathbf{D}^{i} \cdot \mathbf{B} \simeq \mp \mathbf{D}^{i} \cdot \mathbf{z}=\mp \frac{N_{e}-\bar{N}_{e}}{N+\bar{N}}
$$

corresponding to the conservation of the (electron) lepton number, and implying pair conversions of the kind $\nu_{e} \bar{\nu}_{e} \rightarrow \nu_{x} \bar{\nu}_{x}[43]$.

In the special case of (nearly) constant neutrino density $(\dot{\mu} \simeq 0)$, another conserved scalar is the average energy per neutrino pair, given by

$$
\mathcal{E}=\mathbf{B} \cdot(\mathbf{W}+\overline{\mathbf{W}})+\frac{1}{2} \mu \mathbf{D}^{2}=\mathcal{V}+\mathcal{T},
$$

where the first (second) term acts as a sort of potential (kinetic) energy.

When $r$ is sufficiently large to make self-interaction effects vanish $(\mu \ll \omega)$, the kinetic term $\mathcal{T}$ also vanishes, and the "ground state" for $\mathcal{E}$ would corresponds to the minimization of the potential $\mathcal{V}$, i.e., to $\mathbf{W}$ and $\overline{\mathbf{W}}$ aligned as much as possible with -B (in any hierarchy), provided that lepton number is also conserved.

Since the vectors $\mathbf{W}$ and $\overline{\mathbf{W}}$ always start aligned to $\mathbf{z}$, this implies that, in normal hierarchy $(\mathbf{z} \simeq-\mathbf{B})$ they end up in the same position, trivially conserving lepton number (i.e., the system starts - and remains - close to the minimum of the potential energy). Conversely, in inverted hierarchy $(\mathbf{z} \simeq+\mathbf{B})$ the vectors $\mathbf{W}$ and $\overline{\mathbf{W}}$ start antialigned with $-\mathbf{B}$ (maximum of the potential), and for large $r$ they should reverse their direction 
in order to approach the potential minimum. Reversal cannot be complete, however, since it would maximally violate (invert) lepton number. Consistent minimization of the potential can be achieved by complete reversal of the smallest vector $\overline{\mathbf{W}}$, and by partial reversal of the largest vector $\mathbf{W}$ (note that the excess of neutrinos over antineutrinos leads to $|\mathbf{W}|>|\overline{\mathbf{W}}|$ ).

More precisely, only a fraction $\mathbf{W}_{>}$of $\mathbf{W}$, the fraction above a certain critical (split) energy $E_{c}$, is reversed, while the complementary fraction, $\mathbf{W}_{<}=\mathbf{W}-\mathbf{W}_{>}$, remains unaltered [44-49]. In such final state, the critical energy is fixed by lepton number conservation, i.e., by $D_{z}^{i}=D_{z}^{f}$ with

$$
(N+\bar{N}) D_{z}^{f}=\int_{0}^{E_{c}} d E\left(n_{e}-n_{x}\right)-\int_{E_{c}}^{\infty} d E\left(n_{e}-n_{x}\right)+\int_{0}^{\infty} d E\left(\bar{n}_{e}-\bar{n}_{x}\right),
$$

the last two terms having the opposite overall sign in the initial state $(N+\bar{N}) D_{z}^{i}$. Then one gets an implicit equation for $E_{c}$,

$$
\int_{E_{c}}^{\infty} d E\left(n_{e}-n_{x}\right)=\int_{0}^{\infty} d E\left(\bar{n}_{e}-\bar{n}_{x}\right)
$$

which, in our specific SN model, is solved for $E_{c} \simeq 7 \mathrm{MeV}$. When all collective effects are terminated $(\mu \ll \omega)$, one expects a nearly complete inversion of the polarization vectors for $E>E_{c}$, and thus a "stepwise swap" between the $\nu_{e}$ and $\nu_{x}$ energy spectra.

Of course, such reasoning is heuristic, and does not prove that the dynamics allows the system to reach the peculiar final state discussed above. We refer the reader to [4448] for explicit solutions constructed in adiabatic approximation (slowly decreasing $\mu$ ), which indeed lead to spectral split under rather broad assumptions. On the other hand, such adiabatic solutions average out the interesting transient phenomenon of bipolar oscillations $[42,43]$, which we discuss below.

\subsection{Alignment Approximation and Gyroscopic Pendulum}

For $\mu|\mathbf{D}| \gg \omega$, Eqs. (35)-(38) reduce to the same form $\dot{\mathbf{V}} \simeq \mu \mathbf{D} \times \mathbf{V}$, and thus all polarization vectors $\mathbf{V}=\mathbf{P}, \overline{\mathbf{P}}, \mathbf{J}$, and $\overline{\mathbf{J}}$ have the same dynamics (in particular, they remain closely aligned to each other, and to the $z$-axis, as they are at the start). As $\mu$ decreases, the $\pm \omega$ terms in the EOM start to be non-negligible, and neutrino and antineutrino polarization vectors develop different precession histories. As far as $\Delta \omega / \mu$ remains small, where $\Delta \omega$ is the typical energy spread, the individual $\mathbf{P}$ 's stick to the global vector $\mathbf{J}$ (i.e., their components parallel to $\mathbf{J}$ typically dominate over the transverse ones), and analogously the $\overline{\mathbf{P}}$ 's stick to $\overline{\mathbf{J}}$, with $\mathbf{J}$ and $\overline{\mathbf{J}}$ gradually separating from each other.\|

Within such "alignment approximation," also $\mathbf{W}(\overline{\mathbf{W}})$ is nearly parallel to $\mathbf{J}(\overline{\mathbf{J}})$,

$$
\begin{aligned}
& \mathbf{W} \simeq w \mathbf{J}, \\
& \overline{\mathbf{W}} \simeq \bar{w} \overline{\mathbf{J}},
\end{aligned}
$$

$\|$ This approximation may become ill-defined in the symmetric case $n_{e}=\bar{n}_{e}$ (not our case), where the smallness of $|\mathbf{D}|$ makes the condition $\mu|\mathbf{D}| \gg \omega$ critical and the $(\mathbf{J}, \overline{\mathbf{J}})$ separation unclear. See, e.g., the remarks in Sec. VI A of [43]. 
Collective neutrino flavor transitions in supernovae and the role of trajectory averaging 12 and Eqs. (37)-(38) become

$$
\begin{aligned}
& \dot{\mathbf{J}}=\left[\left(\omega_{\text {dif }}+\omega_{\text {ave }}\right) \mathbf{B}+\mu \mathbf{D}\right] \times \mathbf{J}, \\
& \dot{\overline{\mathbf{J}}}=\left[\left(\omega_{\text {dif }}-\omega_{\text {ave }}\right) \mathbf{B}+\mu \mathbf{D}\right] \times \overline{\mathbf{J}},
\end{aligned}
$$

where we have defined

$$
\begin{aligned}
& \omega_{\text {ave }}=(w+\bar{w}) / 2, \\
& \omega_{\text {dif }}=(w-\bar{w}) / 2 .
\end{aligned}
$$

Equations (51)-(52) imply conservation of the vector moduli $J=|\mathbf{J}|$ and $\bar{J}=|\overline{\mathbf{J}}|$, as well as of $W=|\mathbf{W}|$ and $\bar{W}=|\overline{\mathbf{W}}|$. The frequencies $w$ and $\bar{w}$ can be evaluated, e.g., in the initial state, providing

$$
\begin{aligned}
\omega_{\mathrm{ave}} & =\frac{1}{2}\left(\frac{W_{z}^{i}}{J_{z}^{i}}+\frac{\bar{W}_{z}^{i}}{\bar{J}_{z}^{i}}\right)=\frac{\int d E \omega\left(n_{e}-n_{x}\right)}{2\left(N_{e}-N_{x}\right)}+\frac{\int d E \omega\left(\bar{n}_{e}-\bar{n}_{x}\right)}{2\left(\bar{N}_{e}-\bar{N}_{x}\right)} \\
& =\frac{\Delta m^{2} c_{-}}{4}\left(\beta_{e}+\bar{\beta}_{e}+2 \beta_{x}\right) .
\end{aligned}
$$

In our specific SN model, for $\Delta m^{2}=2 \times 10^{-3} \mathrm{eV}^{2}$ it is

$$
\omega_{\text {ave }} \simeq 0.9 \mathrm{~km}^{-1} \text {. }
$$

By going in a co-rotating frame with frequency $\omega_{\text {dif }} \mathbf{B} \simeq \mp \omega_{\text {dif }} \mathbf{z}$, the terms $\omega_{\text {dif }} \mathbf{B} \times$ in Eqs. (51)-(52) are rotated away, with no other formal change in the EOM for $\mathbf{J}$ and $\overline{\mathbf{J}}$.

The $\omega_{\text {dif }} \mathbf{B} \times$ terms disappear also from the EOM of $\mathbf{S}$ and $\mathbf{D}$. By defining $\mathbf{Q}=\mathbf{S}-\left(\omega_{\text {ave }} / \mu\right) \mathbf{B}[43]$, and assuming $\dot{\mu} \simeq 0$ (adiabatic variations of $\mu$ ), Eqs. (39)-(40) can be written as

$$
\begin{aligned}
& \dot{\mathbf{Q}}=\mu \mathbf{D} \times \mathbf{Q}, \\
& \dot{\mathbf{D}}=\omega_{\text {ave }} \mathbf{B} \times \mathbf{Q},
\end{aligned}
$$

showing that the $(\nu, \bar{\nu})$ ensamble is characterized by a single, collective frequency $\omega_{\text {ave }}$, despite the existence of a continuous energy spectra. Notice that $Q=|\mathbf{Q}|$ is conserved, as well as $\mathbf{D} \cdot \mathbf{B}$ and $\mathbf{D} \cdot \mathbf{Q}[43]$.

It has been realized that Eqs. (57)-(58) describe a gyroscopic pendulum in flavor space $[43,44]$. In particular, by making the identifications

$$
\begin{array}{ll}
\mathbf{Q} / Q & \equiv \mathbf{r}(\text { unit length vector }), \\
\mathbf{D} & \equiv \mathbf{L}(\text { total angular momentum }), \\
\mu^{-1} & \equiv m \text { (mass) } \\
\mathbf{D} \cdot \mathbf{Q} / Q & \equiv \sigma(\text { spin }), \\
\omega_{\text {ave }} \mu Q \mathbf{B} & \equiv-\mathbf{g} \text { (gravity field) },
\end{array}
$$

one can write Eqs. (57)-(58) in the form (after right-multiplying Eq. (57) by $\mathbf{r} \times$ )

$$
\begin{aligned}
& \mathbf{L}=m \mathbf{r} \times \dot{\mathbf{r}}+\sigma \mathbf{r}, \\
& \dot{\mathbf{L}}=m \mathbf{r} \times \mathbf{g},
\end{aligned}
$$


which are the equations of motion of a spherical pendulum of unit length $(|\mathbf{r}|=1)$, subject to a constant gravity field $\mathbf{g}$, and characterized by a point-like bob of mass $m$ which spins around the pendulum axis $\mathbf{r}$ with constant (inner) angular momentum $\sigma$. The most general evolution of this system is a combination of two periodic motions of the bob, one around the vertical $\mathbf{g}$ axis (precession) and the other along it (nutation) $[43,44]$. An explicit solution of the EOM can be constructed in terms of quadratures involving the three integrals of motion $[52,53]$, namely, the spin $\sigma=\mathbf{L} \cdot \mathbf{r}$, the vertical component of the angular momentum $\mathbf{L} \cdot \mathbf{g} /|\mathbf{g}|$, and the energy $\mathcal{E}$ which now equals

$$
\mathcal{E}=-m \mathbf{g} \cdot \mathbf{r}+\left(\frac{m}{2} \dot{\mathbf{r}}^{2}+\frac{\sigma^{2}}{2 m}\right),
$$

where we have kept the spin term in the (bracketed) kinetic energy. The explicit solution, however, involves elliptic integrals and is not particularly transparent. Here it is sufficient to recall the following global features of the pendulum motion.

In normal hierarchy, the pendulum starts close to the stable, downward position (the misalignment being of $O\left(\theta_{13}\right)$ ), and remains close to it as $\mu$ slowly decreases (i.e., $m$ slowly increases). Conversely, in inverted hierarchy, the pendulum starts close to the "unstable," upward position. When $\mu$ is large and thus $m$ is small, however, the bob spin $\sigma$ dominates ("fast rotator"), and the pendulum remains precessing in the upward position to conserve angular momentum ("sleeping top") [44,52]. This situation (also dubbed as "synchronization" $[32,43]$ in the SN neutrino context) is stable if the dominant (spin) kinetic term is larger than the maximum excursion of the potential energy [43, 44, 52], namely,

$$
\frac{\sigma^{2}}{2 m}>2 m|\mathbf{g}|
$$

For large $\mu / \omega_{\text {ave }}$ (and thus vertically aligned polarization vectors), this condition translates into $\mu>\mu_{\text {sup }}$, where

$$
\begin{aligned}
\mu_{\mathrm{sup}} \simeq \frac{4 \omega_{\mathrm{ave}} S_{z}^{i}}{\left(D_{z}^{i}\right)^{2}} & =4 \omega_{\mathrm{ave}} \frac{\left(N_{e}+\bar{N}_{e}\right)^{2}-\left(N_{x}+\bar{N}_{x}\right)^{2}}{\left(N_{e}-\bar{N}_{e}\right)^{2}} \\
& =4 \omega_{\mathrm{ave}} \frac{\left(\beta_{e}+\bar{\beta}_{e}\right)^{2}-4 \beta_{x}^{2}}{\left(\beta_{e}-\bar{\beta}_{e}\right)^{2}} .
\end{aligned}
$$

In our reference $\mathrm{SN}$ model, it is

$$
\mu_{\mathrm{sup}} \simeq 75 \omega_{\mathrm{ave}} \simeq 67 \mathrm{~km}^{-1} \text {. }
$$

Conversely, when $\mu<\mu_{\text {sup }}$, any initial misalignment with the "vertical axis" set by $\mathbf{g}$ (i.e., any $\theta_{13} \neq 0$, no matter how small) triggers the first fall of the pendulum and its subsequent nutations (also dubbed as "bipolar oscillations" in the SN neutrino context). If $\mu$ were constant, bipolar oscillations would be exactly periodic. However, the decrease of $\mu$ implies an increase of the pendulum inertia $(m)$; the pendulum never swings back to exactly the same uppermost position, which instead steadily decreases, together with the vertical amplitude of nutations (roughly $\propto \mu^{1 / 2}[43,44]$ ). Bipolar oscillations are then expected to vanish when self-interaction and vacuum effects are 
comparable, and the "alignment approximation" breaks down. More precisely, one may expect this condition to occur when the $\mu$ and $\omega$ terms in the EOM of $\mathbf{J}$ and $\overline{\mathbf{J}}$ are of the same size $\left(\mu \mathbf{D} \cdot \mathbf{B} \sim \omega_{\text {ave }}\right)$, implying $\mu \sim \mu_{\text {inf }}$ with

$$
\mu_{\mathrm{inf}}=\frac{\omega_{\mathrm{ave}}}{D_{z}^{i}}=\omega_{\mathrm{ave}} \frac{N+\bar{N}}{N_{e}-\bar{N}_{e}}=\omega_{\mathrm{ave}} \frac{\beta_{e}+\bar{\beta}_{e}+2 \beta_{x}}{\beta_{e}-\bar{\beta}_{e}}
$$

In our reference $\mathrm{SN}$ model, it is

$$
\mu_{\mathrm{inf}} \simeq 7.5 \omega_{\mathrm{ave}} \simeq 6.7 \mathrm{~km}^{-1} .
$$

The condition $\mu \simeq \mu_{\text {inf }}$ roughly marks the "end" of bipolar oscillations and of collective effects, but not yet of all self-interaction effects. In particular, spectral split effects continue to build up for $\mu \lesssim \mu_{\text {inf }}$, and eventually freeze out for $\mu \ll \omega_{\text {ave }}$. The reason is that the neutrino spectral split requires a separation of the vector $\mathbf{W}$ into parts $\mathbf{W}_{<}$and $\mathbf{W}_{>}$oppositely evolving in the $z$-component. As far as the alignment approximation holds (and thus bipolar oscillations occur), this split cannot fully develop, and should therefore be complete somewhat beyond the bipolar range. Of course, there is no sharp boundary between the two processes: in the range where $\mu \sim \mu_{\text {inf }}$, one should observe a smooth vanishing of bipolar oscillations, and a gradual build-up of the spectral split, through the polarization reversal of neutrinos with $E>E_{c}$, with an associated non-conservation of $\mathbf{W}$ (and of $\mathbf{J}$ ). Summarizing, we expect the following sequence of dominant phenomena, where the radial ranges refer to our reference SN model:

$$
\begin{array}{ll}
\mu \gtrsim \mu_{\text {sup }} & \text { : synchronized oscillations }(r \lesssim 55 \mathrm{~km}), \\
\mu_{\text {inf }} \lesssim \mu \lesssim \mu_{\text {sup }} & : \text { bipolar oscillations }(55 \mathrm{~km} \lesssim r \lesssim 100 \mathrm{~km}), \\
\mu \lesssim \mu_{\text {inf }} & : \text { spectral split }(r \gtrsim 100 \mathrm{~km}) .
\end{array}
$$

Such ranges are explictly shown in Fig. 2. For numerical purposes, we shall stop our investigations to $200 \mathrm{~km}$ in this paper. We are not concerned here with subsequent (ordinary or "stochastic") MSW transitions which may occur later at $r \sim O\left(10^{3}\right) \mathrm{km}$ when $\lambda \simeq \omega$.

\subsection{The Revenge of Matter Effects}

Matter $\lambda$ effects can alter the previous description in two ways: (1) by anticipating the MSW condition $\lambda \simeq \omega_{\text {ave }}$ within the first $O(200) \mathrm{km}$ in shallow matter profiles (not our case), thus interfering with collective effects in the same range $[17,46] ;(2)$ by altering the dynamics when the polarization vectors are very close to $\mathbf{z}$, which occurs just at the transition between synchronized and bipolar oscillations [43]. When the latter transition occurs, matter $(\lambda)$ and small mixing $\left(\theta_{13}\right)$ effects cannot be completely co-rotated away, the $\mathbf{B} \simeq \mp \mathbf{z}$ approximation fails, and the transverse components of $\mathbf{B}$ (oscillating with amplitude $\sin 2 \theta_{13}$ and frequency $\lambda$ ) must be taken into account. In the nontrivial case of inverted hierarchy, it turns out that their general effect is to further stabilize the "upward" pendulum position [43], elongating the period of (at least) the first bipolar swing. Explicit analytic estimates have been presented in [43] for the symmetric case 


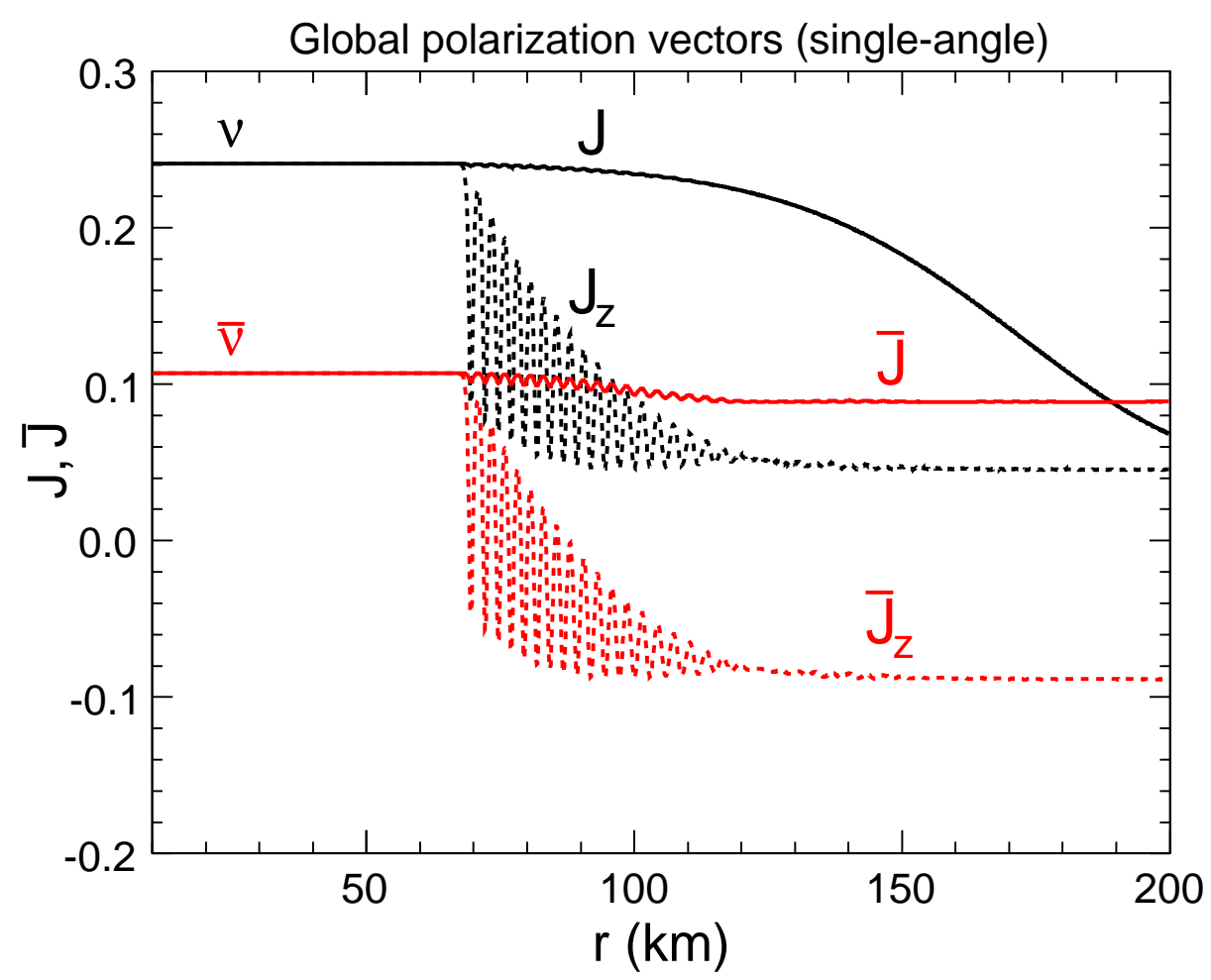

Figure 3. Single-angle simulation in inverted hierarchy: Modulus and $z$-component of the global polarization vector of neutrinos $(\mathbf{J})$ and antineutrinos $(\overline{\mathbf{J}})$, as a function of radius. The difference $D_{z}=J_{z}-\bar{J}_{z}$ (not shown) remains strictly constant in $r$.

$n_{e}=\bar{n}_{e}$, by solving the full EOM with time-varying $\mathbf{B}$ and for small deviations around the vertical directions. If we take these estimates as a reasonable proxy also for our asymmetric case $\left(n_{e} \neq \bar{n}_{e}\right)$, we expect that the first bipolar oscillation (nutation) should be a factor of $\sim 4$ longer than the next ones (which are much less affected by these subtle matter effect, the uppermost pendulum position becoming increasingly tilted for decreasing $\mu$ ). The onset of bipolar oscillations is then expected to be somewhat delayed beyond $r=55 \mathrm{~km}$ in our reference SN model, by a time span equivalent to "a few nutations."

\section{Single-Angle Approximation: Numerical results}

The previous analytical expectations for the single-angle case are nicely confirmed by our simulations. We numerically evolve Eqs. (33)-(34) in the range $r \in[10,200] \mathrm{km}$ within our reference SN model, considering only in the nontrivial case of inverted hierarchy (we have anyway checked that no significant effect occurs in normal hierarchy). Technical details are discussed in the Appendix: here we focus only on the results and their interpretation.

Figure 3 shows the radial evolution of the modulus $J$ and $z$-component $J_{z}$ of the global neutrino polarization vector $\mathbf{J}$ (and analogously for the antineutrino vector $\overline{\mathbf{J}}$ ). 
Their radial profile can be interpreted as follows. Up to $\sim 68 \mathrm{~km}$, it is $J=J_{z}$ and $\bar{J}=\bar{J}_{z}$ : all polarization vectors are "glued" (synchronized) along the vertical axis, and the gyroscopic flavor pendulum just spins in the upward position without falling. At $r \sim 68 \mathrm{~km}$, the pendulum falls for the first time and nutations appear, marking the transition from synchronized to bipolar regime. The transition is retarded by a few nutation periods (from the expected $\sim 55 \mathrm{~km}$ to $\sim 68 \mathrm{~km}$ ) by the matter effects discussed in the previous Section. The nutation amplitude gradually decrease (as $\sim \mu^{1 / 2}$ ), and bipolar oscillations eventually vanish for $r \gtrsim 100 \mathrm{~km}$, as expected.

At the same time, the spectral split effect builds up. Antineutrinos tend to completely reverse the polarization vector $(\overline{\mathbf{J}} \rightarrow-\overline{\mathbf{J}})$, thus minimizing their "potential energy" (after which nothing relevant happens to them), so that $\bar{J}_{z} \simeq-\bar{J}$ asymptotically. $\Phi$ Neutrinos also try to invert their global polarization vector (as much as it is allowed by lepton number conservation) as soon as the alignment approximation breaks down $\left(\mu \lesssim \mu_{\text {inf }}\right)$ and non-conservation of $J$ is allowed. Indeed, for $r \gtrsim 100 \mathrm{~km}$, $J$ decreases. Eventually the situation $J \simeq J_{z}$ is reached (slightly beyond the $r$ range in Fig. 3), when the spectral splitting is frozen, corresponding to a final state with $\mathbf{J}$ aligned with $+\mathbf{z}$ and $\overline{\mathbf{J}}$ aligned with $-\mathbf{z}$. In all the above processes, lepton number is strictly conserved, leading to the constancy of $D_{z}=J_{z}-\bar{J}_{z}$ at any $r$. From the point of view of observable oscillation probabilities (related to the $z$-component of polarization vectors), the situation is basically frozen well before $r \sim 200 \mathrm{~km}$. We can conclude that the behavior of $J, \bar{J}, J_{z}$ and $\bar{J}_{z}$ is well understood, with good agreement between analytical expectations and numerical simulations. The agreement can also be extended to more detailed features of the energy spectrum, as discussed next.

Figure 4 shows the behavior of the individual polarization components $P_{z}$ (upper panel) and $\bar{P}_{z}$ (lower panel) as a function of $r$, for five representative values of energy (in $\mathrm{MeV}$ ): $E_{1} \simeq 5.2, E_{2} \simeq 12.4, E_{3} \simeq 19.1, E_{4} \simeq 23.8$, and $E_{5} \simeq 31.3$ (which are a subset of the grid sampling energies, hence their "non-rounded" values). ${ }^{+}$In Fig. 4, the onset of bipolar oscillations and their nutation periods are clearly equal for both $\nu$ and $\bar{\nu}$ at any energy, confirming the appearance of a self-induced collective behavior, characterized by a single frequency parameter $\omega_{\text {ave }}$ for all (anti)neutrinos, despite the spread of vacuum oscillation frequencies $\omega$. However, the fate of each $P_{z}$ or $\bar{P}_{z}$ does depend on energy. For neutrinos (upper panel in Fig. 4), one can clearly see the phenomenon of spectral split around $E_{c} \simeq 7 \mathrm{MeV}$ : the curve at $E_{1}<E_{c}$ ends up at the same initial value, while the curves at $E_{2}, E_{4}, E_{5}>E_{c}$ show the expected inversion $P_{z} \rightarrow-P_{z}$. Only the curve at energy $E_{3}$ does not change much $\left(P_{z} \simeq 0\right.$ at any $r$ ), being close to the energy $E_{\text {eq }}$ where the $\nu_{e}$ and $\nu_{x}$ fluxes are equal (see Fig. 1), and flavor transformations are inoperative. For antineutrinos (lower panel in Fig. 4), all curves show complete polarization reversal as expected $\left(\bar{P}_{z} \rightarrow-\bar{P}_{z}\right)$, including the "trivial" case $\bar{P}_{z} \simeq 0$ at $E_{4} \simeq \bar{E}_{\text {eq }}$, where the $\bar{\nu}_{e}$ and $\bar{\nu}_{x}$ fluxes are equal. We conclude that the numerical simulations confirm the

- There is actually a slight loss of $\bar{J}$ during bipolar oscillations in Fig. 3 (as also numerically observed in [49]) which makes the final $\left|J_{z}^{f}\right| \simeq 0.09$ slightly smaller than the initial $J_{z}^{i} \simeq 0.1$.

+ We do not show the moduli, which are always strictly conserved. 
Collective neutrino flavor transitions in supernovae and the role of trajectory averaging 17

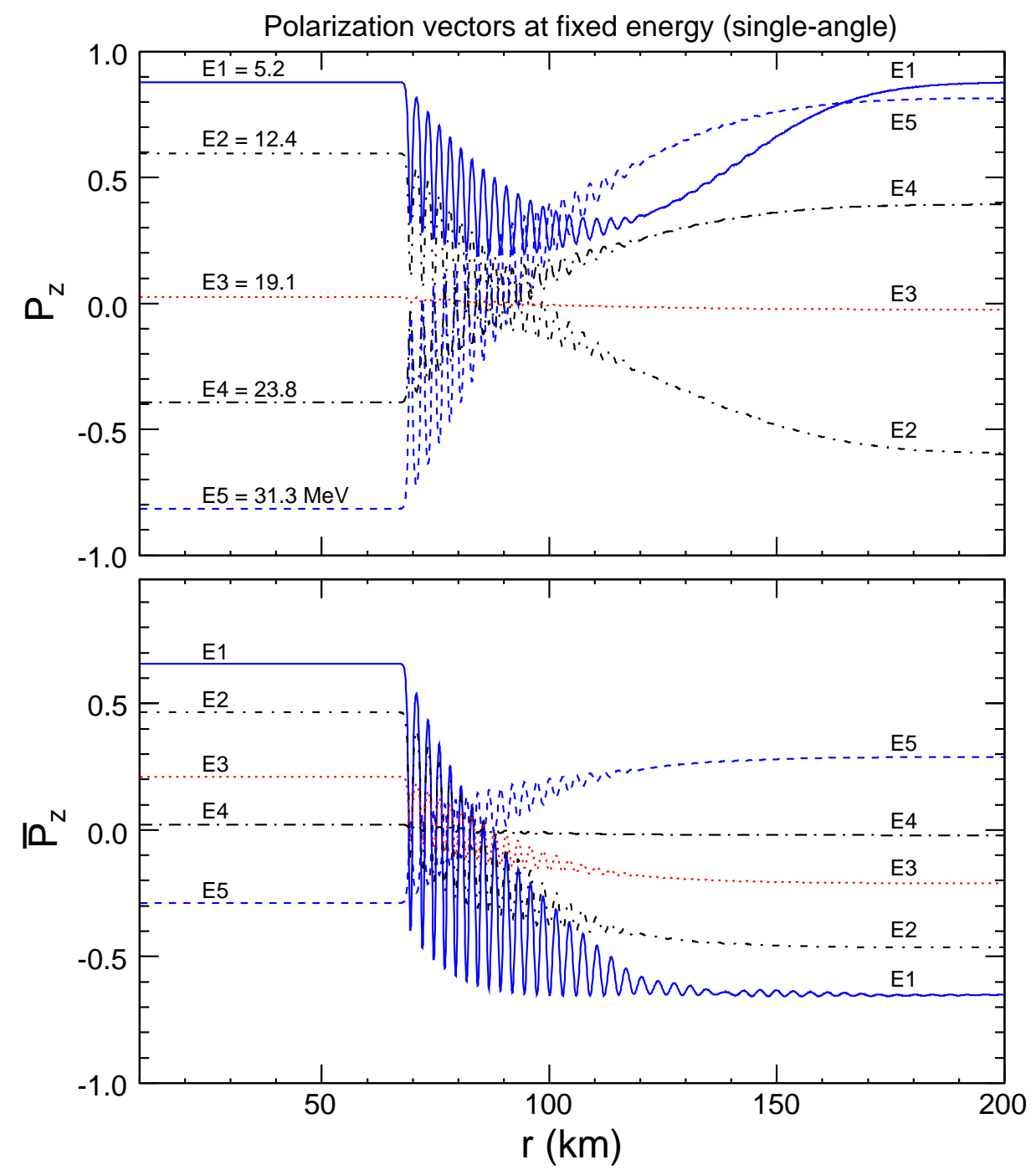

Figure 4. Single-angle simulation in inverted hierarchy: $z$-component of the polarization vector of neutrinos $\left(P_{z}\right.$, upper panel $)$ and antineutrinos $\left(\bar{P}_{z}\right.$, lower panel) as a function of radius, for five representative values of the energy.

end of bipolar oscillations and the appearance of the energy split phenomenon (around $r \sim 100 \mathrm{~km})$ with the expected global features. There is only a minor "unexpected" effect (lack of $\bar{P}_{z}$ reversal for $E \lesssim 4 \mathrm{MeV}$, not shown in Fig. 4), as commented below.

At $r=200 \mathrm{~km}$, self-induced flavor transformations have basically ended. The transition from initial $(r=10 \mathrm{~km})$ to final $(r=200 \mathrm{~km})$ fluxes implies, in our twofamily scenario $\left(P_{e e}=P_{x x}=1-P_{e x}\right.$, see also the remarks at the beginning of Sec. 2),

$$
\begin{aligned}
& \frac{\phi_{e}^{i}(E)}{\left\langle E_{e}\right\rangle} \longrightarrow \frac{\phi_{e}^{i}(E)}{\left\langle E_{e}\right\rangle} P_{e e}(E)+\frac{\phi_{x}^{i}(E)}{\left\langle E_{x}\right\rangle}\left[1-P_{e e}(E)\right], \\
& \frac{\phi_{x}^{i}(E)}{\left\langle E_{x}\right\rangle} \longrightarrow \frac{\phi_{x}^{i}(E)}{\left\langle E_{x}\right\rangle} P_{e e}(E)+\frac{\phi_{e}^{i}(E)}{\left\langle E_{e}\right\rangle}\left[1-P_{e e}(E)\right],
\end{aligned}
$$

and similarly for antineutrinos. 

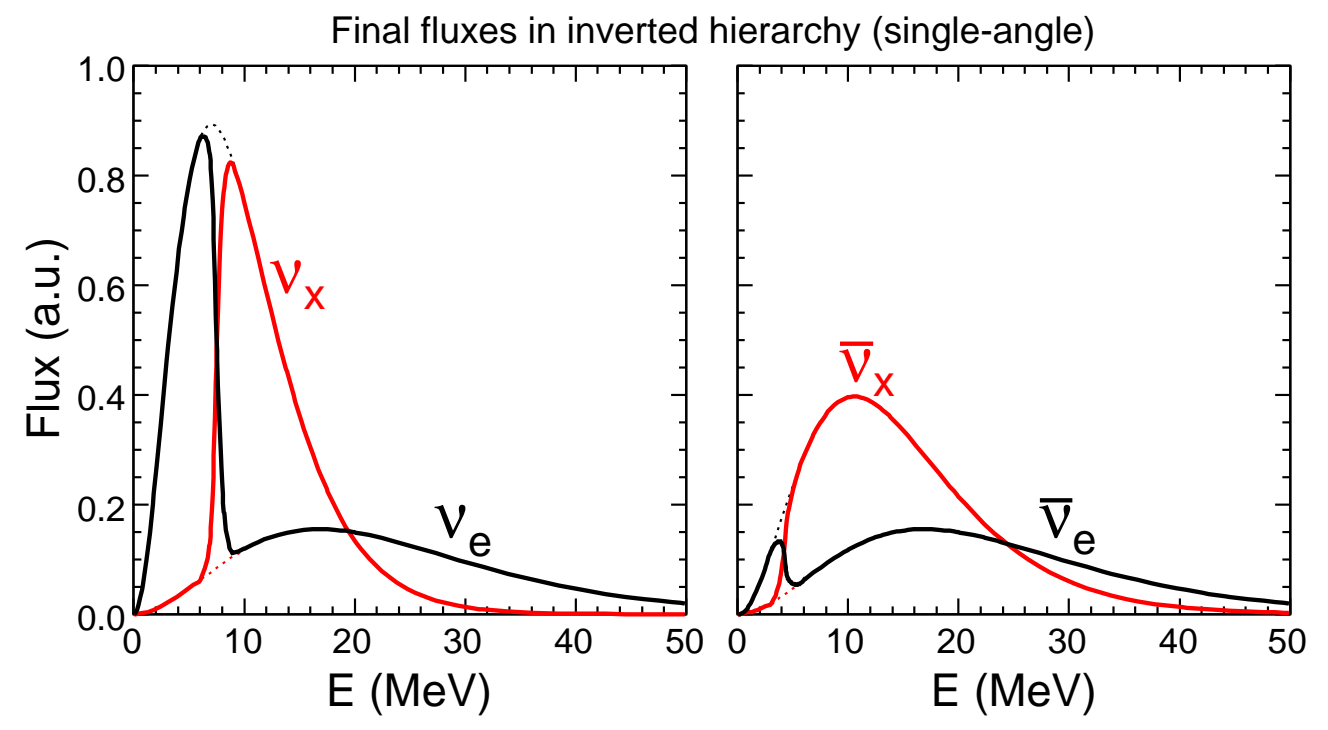

Figure 5. Single-angle simulation in inverted hierarchy: Final fluxes (at $r=200 \mathrm{~km}$, in arbitrary units) for different neutrino species as a function of energy. Initial fluxes are shown as dotted lines to guide the eye.

Figure 5 shows the final neutrino and antineutrino fluxes calculated in this way. The left panel (neutrinos) clearly shows the spectral split effect, and the corresponding sudden swap of $\nu_{e}$ and $\nu_{x}$ fluxes above $E_{c} \simeq 7 \mathrm{MeV}$. In the right panel of Fig. 5, the final antineutrino spectra are basically completely swapped with respect to the initial ones (compare with Fig. 1), except at very low energies, where there appears an "antineutrino" spectral split. We relate this phenomenon to the loss of $\bar{J}$ and of $\left|\bar{J}_{z}\right|$ observed and commented in Fig. 3: The small deficit $\left|\bar{J}_{z}^{f}\right|<\bar{J}_{z}^{i}$ can indeed obtained, analogously to the neutrino case, through the lack of $\bar{P}_{z}$ reversal for low-energy antineutrinos $\left(E<\bar{E}_{c}\right.$ with $\left.\bar{E}_{c} \lesssim 4 \mathrm{MeV}\right)$. A better understanding of this minor feature and of the $\left|\bar{J}_{z}\right|$ loss would be desirable; however, we anticipate that the antineutrino spectral split is largely smeared out in multi-angle simulation, contrary to the neutrino spectral split which appears to be a robust, observable feature.

\section{Multi-angle Simulations: Notation and Numerical results}

In the multi-angle case (applied to the neutrino bulb model), any single polarization vector depends not only on the energy $E$ and on the total propagation distance along a neutrino trajectory $t$, but also on the (incident) angle $\vartheta$ between the neutrino trajectory and the polar axis. The vectors $\mathbf{P}_{\vartheta}(E, t)$ and $\overline{\mathbf{P}}_{\vartheta}(E, t)$ obey then the following EOM [17],

$$
\begin{aligned}
& \dot{\mathbf{P}}_{\vartheta}=\left[+\omega \mathbf{B}+\lambda \mathbf{z}+2 \pi \sqrt{2} G_{F} \int d c_{\vartheta^{\prime}} d E\left(1-c_{\vartheta} c_{\vartheta^{\prime}}\right)\left(j \mathbf{P}_{\vartheta^{\prime}}-\bar{j} \overline{\mathbf{P}}_{\vartheta^{\prime}}\right)\right] \times \mathbf{P}_{\vartheta}, \\
& \dot{\overline{\mathbf{P}}}_{\vartheta}=\left[-\omega \mathbf{B}+\lambda \mathbf{z}+2 \pi \sqrt{2} G_{F} \int d c_{\vartheta^{\prime}} d E\left(1-c_{\vartheta} c_{\vartheta^{\prime}}\right)\left(j \mathbf{P}_{\vartheta^{\prime}}-\bar{j} \overline{\mathbf{P}}_{\vartheta^{\prime}}\right)\right] \times \overline{\mathbf{P}}_{\vartheta},
\end{aligned}
$$


Collective neutrino flavor transitions in supernovae and the role of trajectory averaging 19

where $c_{\vartheta}=\cos \vartheta$ with $\vartheta \in\left[0, \vartheta_{\max }\right]$ and $c_{\vartheta^{\prime}}=\cos \vartheta^{\prime} \in\left[\cos \vartheta_{\max }, 1\right]{ }^{*}$ while $j$ and $\bar{j}$ are the total neutrino and antineutrino number densities

$$
\begin{aligned}
& j=j_{e}+j_{x}, \\
& \bar{j}=\bar{j}_{e}+\bar{j}_{x},
\end{aligned}
$$

as defined in Sec. 2.2.

It is useful to characterize all the properties of the neutrino beam by using the radius $r$ and the emission angle $\vartheta_{0}$ at the neutrinosphere [17], by means of

$$
\begin{aligned}
r \sin \vartheta & =R_{\nu} \sin \vartheta_{0}, \\
t & =\sqrt{r^{2}-R_{\nu}^{2} \sin ^{2} \vartheta_{0}}-R_{\nu} \cos \vartheta_{0},
\end{aligned}
$$

the range of $\cos \vartheta_{0}$ being constantly $[0,1]$ at any $r$. Along a generic trajectory at angle $\vartheta$, $d t=d r / c_{\vartheta}$, and thus $d_{t}=c_{\vartheta} d_{r}$. Since any couple $(t, \vartheta)$ is in one-to-one correspondence with $\left(r, \vartheta_{0}\right)$, the polarization vectors can be relabeled as $\mathbf{P}_{\vartheta_{0}}(E, r)$.

For convenience, one may define effective densities $n$ as in the single-angle case, $n(r, E)=2 \pi D(r) j(E)$, so that the initial conditions at $r=R_{\nu}$ become as usual

$$
\begin{aligned}
& \mathbf{P}_{\vartheta_{0}}^{i}=\left(j_{e}-j_{x}\right) / j=\left(n_{e}-n_{x}\right) / n, \\
& \overline{\mathbf{P}}_{\vartheta_{0}}^{i}=\left(\bar{j}_{e}-\bar{j}_{x}\right) / \bar{j}=\left(\bar{n}_{e}-\bar{n}_{x}\right) / \bar{n} .
\end{aligned}
$$

The single-angle limit is recovered by fixing $\vartheta=0=\vartheta_{0}$ and by assuming that all polarization vectors behave as the ones at $\vartheta_{0}=0$, in which case the integral $\int d c_{\vartheta^{\prime}}\left(1-c_{\vartheta^{\prime}}\right)=D(r)$ is factorized out (this is actually the way $D(r)$ is originally defined [17]). $\sharp$

Equations (77)-(78) reduce then to Eqs. (33)-(34) after the integrated densities $N$ and $\bar{N}$ are introduced as in Sec. 2.2 .

Angle-averaged polarization vectors can be defined as

$$
\mathbf{P}=\frac{\int d c_{\vartheta_{0}} c_{\vartheta_{0}} \mathbf{P}_{\vartheta_{0}}}{\int d c_{\vartheta_{0}} c_{\vartheta_{0}}}
$$

(and similarly for $\overline{\mathbf{P}}$ ), where the "extra" cosine factor accounts for projection in radial direction [49]. Global polarization vectors $\mathbf{J}, \overline{\mathbf{J}}$ and $\mathbf{D}=\mathbf{J}-\overline{\mathbf{J}}$ can then be defined in the same way as in Sec. 3.2. Although their EOM are not as simple as in the single-angle case, it turns out that $\mathbf{D} \cdot \mathbf{B} \simeq D_{z}$ is still a conserved scalar [43,49] (proof omitted).

The angular dependence of the neutrino-neutrino interaction strength is generally expected to introduce some "dephasing" or kinematical decoherence between different neutrino trajectories, and to smear out the "fine structures" observed in single-angle simulations. While decoherence effects are dominant in the symmetric case $\left(n_{e}=\bar{n}_{e}\right)$ [34], they seem to be only subdominant in asymmetric cases $\left(n_{e} \neq \bar{n}_{e}\right)[17,49]$. In the latter case their quantitative description lacks, at the moment, of an analytical

* The angles $\vartheta$ and $\vartheta^{\prime}$ must lie in the cone subtending the neutrinosphere. The factor $2 \pi=\int d \varphi$ comes from cylindrical symmetry within this cone.

$\#$ An alternative single-angle case has been recently studied in [49], by selecting the emission angle $\vartheta_{0}=\pi / 4$ instead of $\vartheta_{0}=0=\vartheta$. 


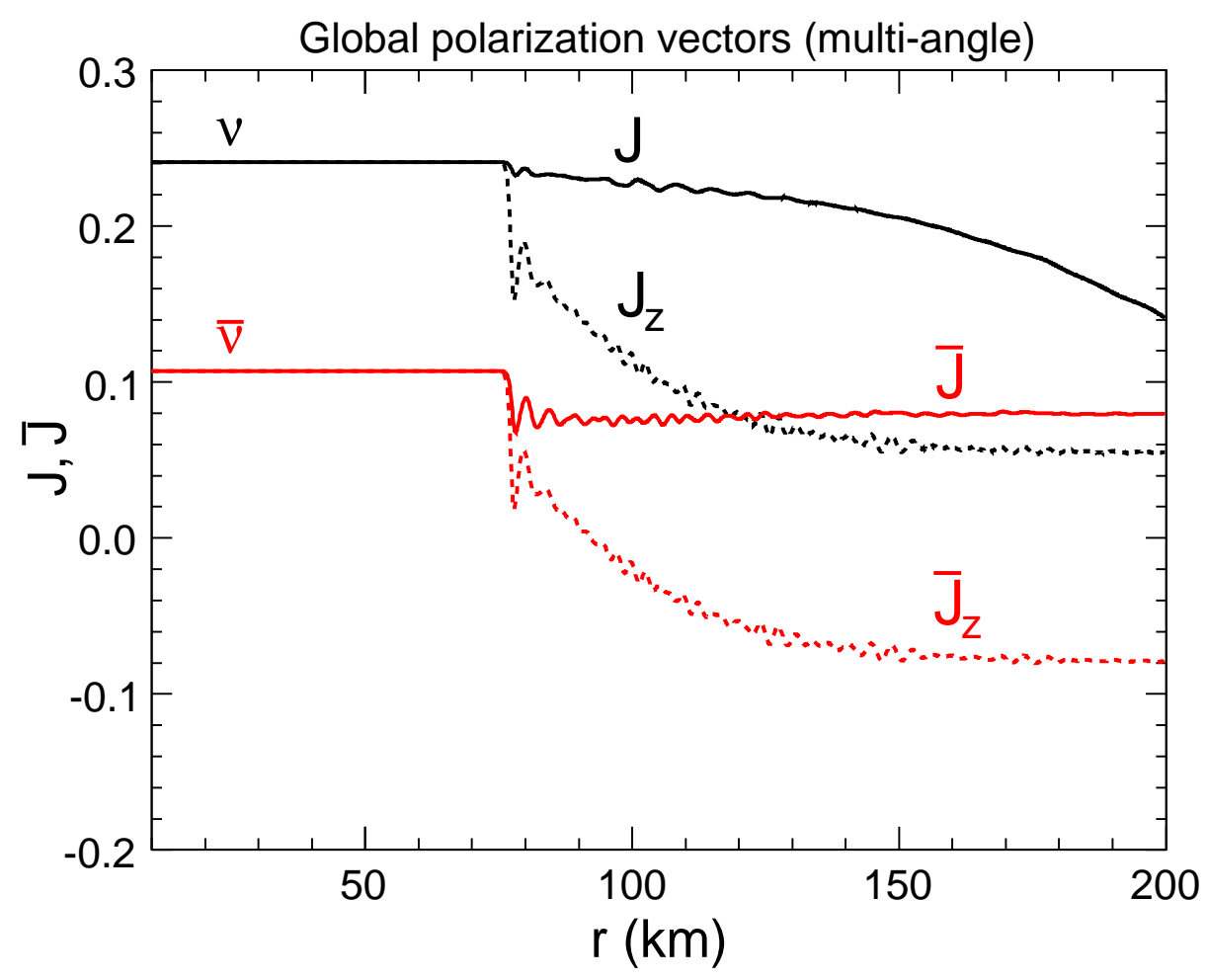

Figure 6. Multi-angle simulation in inverted hierarchy: Modulus and $z$-component of the global polarization vector of neutrinos $(\mathbf{J})$ and antineutrinos $(\overline{\mathbf{J}})$, as a function of radius. The difference $D_{z}=J_{z}-\bar{J}_{z}$ (not shown) remains strictly constant in $r$.

understanding, and relies mainly on numerical simulations [49]. However, one may at least expect that the appearance of the neutrino spectral split phenomenon is not spoiled in multi-angle cases, being based on the broad-brush picture of potential energy minimization (i.e., final (anti)alignment with $\mathbf{B}$ ) constrained by lepton number conservation (i.e., constant $D_{z}$ ). This expectation is confirmed by the numerical simulations discussed below, which refer only to the nontrivial case of inverted hierarchy.

Figure 6 is the multi-angle analogue of Fig. 3. By comparing the two figures, it appears that bipolar oscillations of $\mathbf{J}$ and $\overline{\mathbf{J}}$ are largely suppressed in the multiangle case, only the first nutation being clearly visible. Moreover, such nutation starts somewhat later $(r \simeq 76 \mathrm{~km})$ as compared with the single-angle case $(r \simeq 68 \mathrm{~km})$. These features can be understood in terms of the different self-interaction effects experienced along different trajectories. In multi-angle simulations, neutrino-neutrino angles can be larger than the (single-angle) average one, leading to somewhat stronger self-interaction effects, which keep the system in synchronized mode for a slightly longer time, and thus delay the first nutation. Along different trajectories, the subsequent bipolar oscillations have also somewhat different amplitudes and phases, which tend to cancel out in the global polarization vectors. For a similar reason (relatively stronger self-interaction effects, as compared to single-angle), in Fig. 6 there is a slightly more pronounced loss (depolarization) of $\bar{J}$ at the start of the first bipolar oscillation, and at the same time a 
Collective neutrino flavor transitions in supernovae and the role of trajectory averaging 21

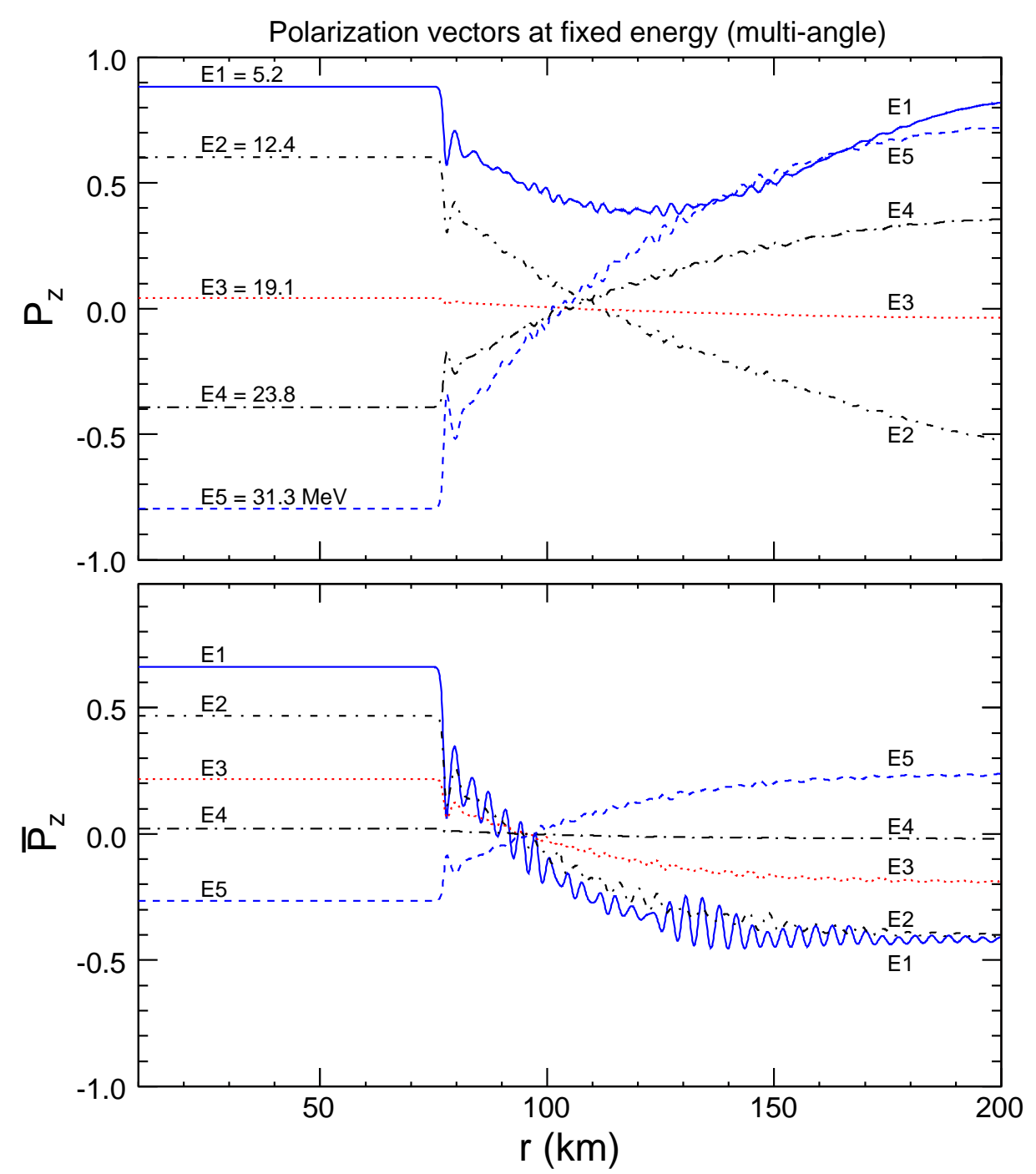

Figure 7. Multi-angle simulation in inverted hierarchy: $z$-component of the polarization vector of neutrinos $\left(P_{z}\right.$, upper panel) and antineutrinos $\left(\bar{P}_{z}\right.$, lower panel) as a function of radius, for five representative values of the energy.

prolonged "coherence" of $\mathbf{J}$ (slower decrease of $J$ ), with respect to Fig. 3. However, just as in the single-angle case, it turns out that $\bar{J}_{z}$ gets finally reversed, while the difference $D_{z}=J_{z}-\bar{J}_{z}$ is exactly conserved. The reversal becomes more evident by looking at specific (anti)neutrino energies.

Figure 7 shows the behavior of the $z$-component of angle-averaged polarization vectors [Eq. (85)] for neutrinos $\left(P_{z}\right.$, upper panel) and antineutrinos $\left(\bar{P}_{z}\right.$, lower panel $)$ in our multi-angle simulation, at fixed energies. The behavior is qualitatively similar to a "smeared version" of the single-angle curves in Fig. 4, with polarization vectors reversing (or not) their $z$-components as expected. The small depth (or absence) of nutations makes it more evident that the polarization reversal (i.e., the spectral split) starts to dominate over the bipolar mode around the expected radius $r \simeq 100 \mathrm{~km}$. 


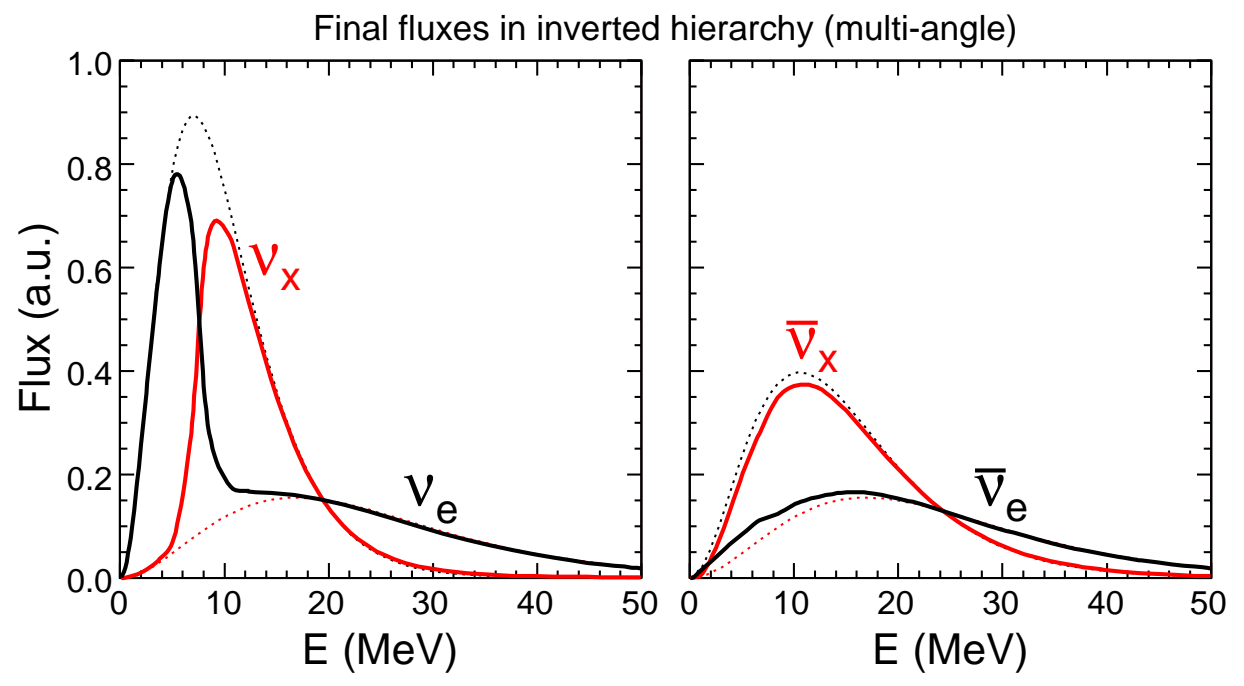

Figure 8. Multi-angle simulation in inverted hierarchy: Final fluxes (at $r=200 \mathrm{~km}$, in arbitrary units) for different neutrino species as a function of energy. Initial fluxes are shown as dotted lines to guide the eye.

Figure 8 shows the final $(r=200 \mathrm{~km}) \nu$ and $\bar{\nu}$ fluxes as a function of energy. The neutrino spectral swap at $E>E_{c} \simeq 7 \mathrm{MeV}$ is rather evident in the left panel, although it is less sharp with respect to the single-angle case in Fig. 5. In the right panel of Fig. 8, the minor feature associated to the "antineutrino spectral split" is largely smeared out (see the same panel in Fig. 5), and survives as a small excess of $\bar{\nu}_{e}$ at low energy.

The spectra in Figure 8 are largely independent from the specific mixing value chosen for the simulations $\left(\sin ^{2} \theta_{13}=10^{-4}\right.$ ), as far as $\theta_{13}>0$ (as we have also checked numerically). Variations of $\sin ^{2} \theta_{13}$ only lead to logarithmic variations in the (unobservable) synchronized-bipolar transition radius, and in the depth of bipolar oscillations $[43,44]$, which are anyway smeared out in multi-angle simulations, as we have just seen. Therefore, the spectra in Figure 8 may be taken as rather general "initial conditions" for possible later (ordinary or stochastic) matter effects, occurring when $\omega \sim \lambda(r)$ at $r \gg 200 \mathrm{~km}$. These later, ordinary matter effects are instead strongly dependent on $\theta_{13}$, and vanish for, say, $\sin ^{2} \theta_{13} \lesssim 10^{-5}$ (see, e.g., [7]). If $\theta_{13}$ is indeed that small (but nonzero), neutrino self-interaction effects could be the only source of flavor transformations in (anti)neutrino spectra.

In conclusion, for $0<\sin ^{2} \theta_{13} \lesssim 10^{-5}$, the observable spectra at the SN exit would be similar to those in Fig. 1 for the normal hierarchy case (no significant flavor transformations of any kind), and to those in Fig. 8 for the inverted hierarchy case (large self-interaction effects). For $\sin ^{2} \theta_{13} \gtrsim 10^{-5}$, the same spectra should be taken as "initial conditions" for the calculation of subsequent MSW effects. Once more, we remark that the decoupling of self-interaction and MSW effects is a characteristic of our adopted SN model, inspired by shock-wave simulations [7]. The phenomenology becomes more complicated in alternative models with shallow matter profiles, when both effects can occur in the same region, as in the simulations performed in $[17,47]$. 
Collective neutrino flavor transitions in supernovae and the role of trajectory averaging 23

\section{Conclusions and prospects}

Neutrino-neutrino interactions in the high-density region near a supernova core have been recently recognized to produce surprising collective effects. Motivated by these developments, we have investigated neutrino flavor transformations in a SN model, where the main self-interaction effects (synchronization, bipolar oscillations, and spectral split) develop well before possible MSW effects.

The neutrino-neutrino interaction strength depends on the intersection angle of their trajectories. Averaging this (variable) angle along a single, representative radial trajectory leads to the so-called single-angle approximation, which allows both elegant analytical insights $[43,44]$ and easier numerical calculations. However, removal of this approximation is needed (through multi-angle simulations) in order to validate the analytical insights, and to show that the main effects are not spoiled by kinematical decoherence. Moreover, many self-interaction effects have actually been first seen numerically and then interpreted analytically a posteriori.

We have thus performed numerical simulations in both single- and multi-angle cases, using continuous energy spectra with significant $\nu-\bar{\nu}$ and $\nu_{e}-\nu_{x}$ asymmetry. The singleangle results can be understood analytically to a large extent, and their main observable effect - in the nontrivial case of inverted hierarchy - is the swap of energy spectra above a critical energy dictated by lepton number conservation [45]. In multi-angle simulations, we find that the "fine structure" of self-interaction effects (e.g., bipolar oscillations) is smeared out, but the spectral swap remains a robust, observable feature. In this sense, trajectory averaging does not play a crucial role. This is the main result of our work.

The swapping of the $\bar{\nu}_{e}$ and $\bar{\nu}_{\mu}$ (as well as of the $\nu_{e}$ and $\nu_{\mu}$ ) fluxes could have an impact on r-process nucleosynthesis $[35-37,50]$, on the energy transfer to the stalling shock wave [54], and on the possibility to observe shock-wave propagation effects in neutrinos. It is also worth studying possible self-interaction effects in the phenomenology of SN 1987A neutrino events [55-57] and of the diffuse supernova neutrino background spectrum $[58,59]$. Further analytical and numerical developments may require to solve the neutrino evolution equations in the complete $3 \nu$ flavor scenario, where new effects associated to the "solar" $\delta m^{2}$ can occur; a recent example has been worked out in [60]. Perturbations of the (cylindrically symmetric) bulb model for neutrino emission might also be considered in more advanced simulations. Finally, there is a continuing interest in more formal aspects of the mean-field approach to neutrino self-interaction effects [61], which has been implicitly assumed in most of the related literature (including this work); see [62] for a recent discussion of its validity and inherent approximations.

In conclusion, twenty years after the SN1987A, the understanding of $\mathrm{SN} \nu$ flavor transformations is still in progress, and surprising self-interaction effects are emerging as possible dominant phenomena. These effects are changing the current paradigm of SN neutrino physics, and demand further analytical and numerical investigations, as well as new experimental inputs and guidance - should Nature be so kind to make a galactic supernova explode. 
Collective neutrino flavor transitions in supernovae and the role of trajectory averaging 24

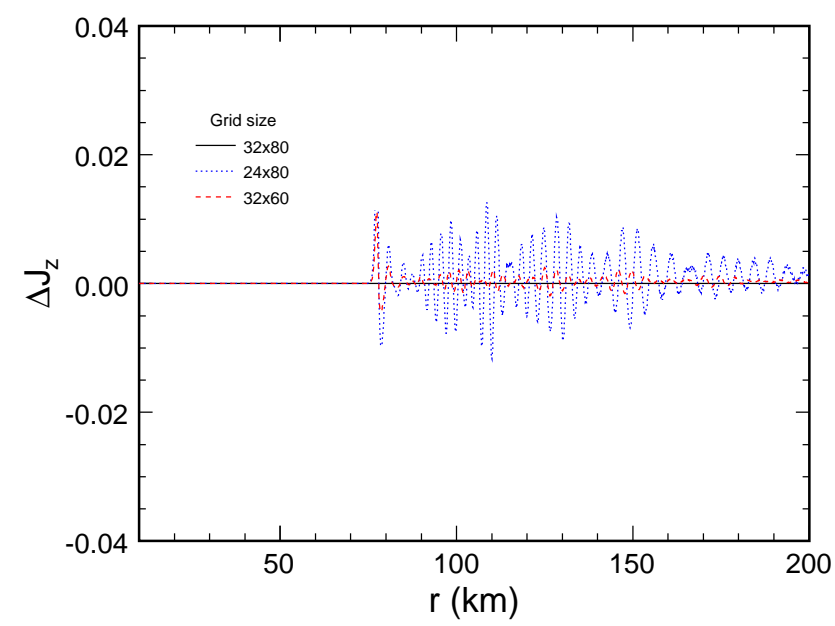

Figure 9. Absolute difference between numerical evaluations of $J_{z}(r)$ in multi-angle simulations, using various (energy) $\times($ angle) grid sizes: $32 \times 80$ (baseline), $32 \times 60$ (dashed), and $24 \times 80$ (dotted).

\section{Acknowledgments}

This work is supported in part by the Italian "Istituto Nazionale di Fisica Nucleare" (INFN) and by the "Ministero dell'Università e della Ricerca" (MiUR) through the "Astroparticle Physics" research project. In Germany, the work of A.M. is supported by an Alexander von Humboldt fellowship grant.

We thank F. Iavernaro and F. Mazzia for invaluable help in implementing their GAMD software [63]. A.M. thanks G.G. Raffelt for illuminating discussions. We acknowledge D. Montanino and G.G. Raffelt for reading the manuscript and for useful remarks, and B. Dasgupta, H. Duan, G.M. Fuller, and C. Lunardini for communications.

\section{Appendix}

We discuss here a few technical aspects of multi-angle numerical simulations, which are much more challenging than single-angle ones. Equations $(77,78)$, after the proper transformation $\vartheta \rightarrow \vartheta_{0}$, provide a set of $6 \times N_{E} \times N_{\vartheta_{0}}$ ordinary differential equations (ODE) in $r$ (in the real domain), where $N_{E}$ and $N_{\vartheta_{0}}$ are the number of points sampling the (anti)neutrino energy $E$ and emission angle $\operatorname{cosine} \cos \vartheta_{0}$, respectively.

This ODE set is stiff, and needs appropriate routines to be solved numerically. After a careful comparison of public routines, we have adopted the GAMD software [63], implemented on a $N_{E} \times N_{\vartheta_{0}}=32 \times 80$ grid. Denser sampling in $\cos \vartheta_{0}$ is required, since the polarization vectors generally vary more rapidly in $\vartheta_{0}$ than in $E$. An exception would be provided by MSW effects interfering with self-interaction ones [17] (not our case), which requires much denser sampling, especially in $E$, in order to track the MSW resonant behavior. In our scenario with no MSW interference, we obtain satisfactory numerical convergence with $N_{E} \times N_{\vartheta_{0}}=32 \times 80$. Figure 9 compares the last three 
steps in a trial sequence with increasingly denser grids $N_{E} \times N_{\vartheta_{0}}$, showing that the final absolute error on the reference quantity $J_{z}$ can be safely estimated to be $<10^{-2}$.

The grid points are not chosen to be equally spaced, but are instead fixed by imposing weighted Gaussian quadrature of the double integrals in the right-hand-side of Eqs. $(77,78)$. In other words, the $N_{E} \times N_{\vartheta_{0}}$ grid points not only sample the energy and angular evolution of the polarization vectors in the ODE set, but are also used to perform the inner Gaussian integrations at each evolutionary step. This "trick" saves a lot of computer time. Nevertheless, a typical simulation over a $32 \times 80$ grid takes $\sim 400$ hours on our local computer facility (a cluster of four, 64-bit and 4Gb RAM processors at $2.4 \mathrm{GHz}$, with Fortran 90 codes running on a Linux platform). We plan to use more powerful (remote) facilities in future works on the subject.

\section{References}

[1] G. G. Raffelt, "Supernova neutrino observations: What can we learn?," astro-ph/0701677.

[2] F. Cavanna, M. L. Costantini, O. Palamara and F. Vissani, "Neutrinos as astrophysical probes," Surveys High Energ. Phys. 19, 35 (2004) [astro-ph/0311256].

[3] L. Wolfenstein, "Neutrino Oscillations In Matter," Phys. Rev. D 17, 2369 (1978); S. P. Mikheev and A. Yu. Smirnov, "Resonance Enhancement Of Oscillations In Matter And Solar Neutrino Spectroscopy," Yad. Fiz. 42, 1441 (1985) [Sov. J. Nucl. Phys. 42, 913 (1985)].

[4] R. C. Schirato and G. M. Fuller, "Connection between supernova shocks, flavor transformation, and the neutrino signal," astro-ph/0205390 (unpublished).

[5] K. Takahashi, K. Sato, H. E. Dalhed and J. R. Wilson, "Shock propagation and neutrino oscillation in supernova," Astropart. Phys. 20, 189 (2003) [astro-ph/0212195].

[6] C. Lunardini and A. Yu. Smirnov, "Probing the neutrino mass hierarchy and the 13-mixing with supernovae," JCAP 0306, 009 (2003) [hep-ph/0302033].

[7] G. L. Fogli, E. Lisi, A. Mirizzi, and D. Montanino, "Analysis of energy- and time-dependence of supernova shock effects on neutrino crossing probabilities," Phys. Rev. D 68, 033005 (2003) [hep-ph/0304056].

[8] R. Tomàs, M. Kachelrieß, G. Raffelt, A. Dighe, H. T. Janka and L. Scheck, "Neutrino signatures of supernova shock and reverse shock propagation," JCAP 0409, 015 (2004) [astro-ph/0407132].

[9] G. L. Fogli, E. Lisi, A. Mirizzi and D. Montanino, "Probing supernova shock waves and neutrino flavor transitions in next-generation water-Cherenkov detectors," JCAP 0504, 002 (2005) [hep$\mathrm{ph} / 0412046]$.

[10] V. Barger, P. Huber and D. Marfatia, "Supernova neutrinos can tell us the neutrino mass hierarchy independently of flux models," Phys. Lett. B 617, 167 (2005) [hep-ph/0501184].

[11] S. Choubey, N. P. Harries and G. G. Ross, "Probing neutrino oscillations from supernovae shock waves via the IceCube detector," Phys. Rev. D 74, 053010 (2006) [hep-ph/0605255].

[12] B. Dasgupta and A. Dighe, "Phase effects in neutrino conversions during a supernova shock wave," Phys. Rev. D 75, 093002 (2007) [hep-ph/0510219].

[13] J. P. Kneller, G. C. McLaughlin and J. Brockman, "Oscillation Effects and Time Variation of the Supernova Neutrino Signal," arXiv:0705.3835 [astro-ph].

[14] G. L. Fogli, E. Lisi, A. Mirizzi and D. Montanino, "Damping of supernova neutrino transitions in stochastic shock-wave density profiles," JCAP 0606, 012 (2006) [hep-ph/0603033].

[15] A. Friedland and A. Gruzinov, "Neutrino signatures of supernova turbulence," astro-ph/0607244.

[16] S. Choubey, N. P. Harries and G. G. Ross, "Turbulent supernova shock waves and the sterile neutrino signature in megaton water detectors," Phys. Rev. D 76, 073013 (2007) [hep$\mathrm{ph} / 0703092]$.

[17] H. Duan, G. M. Fuller, J. Carlson and Y. Z. Qian, "Simulation of coherent non-linear neutrino 
Collective neutrino flavor transitions in supernovae and the role of trajectory averaging 26

flavor transformation in the supernova environment. I: Correlated neutrino trajectories," Phys. Rev. D 74, 105014 (2006) [astro-ph/0606616].

[18] G.M. Fuller, R.W. Mayle, J.R. Wilson, and D.N. Schramm, "Resonant neutrino oscillations and stellar collapse," Astrophys. J. 322, 795 (1987).

[19] J. Pantaleone, "Neutrino oscillations at high densities," Phys. Lett. B 287, 128 (1992).

[20] S. Samuel, "Neutrino oscillations in dense neutrino gases," Phys. Rev. D 48, 1462 (1993).

[21] V. A. Kostelecký, J. Pantaleone and S. Samuel, "Neutrino oscillation in the early universe," Phys. Lett. B 315, 46 (1993).

[22] V. A. Kostelecký and S. Samuel, "Neutrino oscillations in the early universe with an inverted neutrino mass hierarchy," Phys. Lett. B 318, 127 (1993).

[23] V. A. Kostelecký and S. Samuel, "Nonlinear neutrino oscillations in the expanding universe," Phys. Rev. D 49, 1740 (1994).

[24] V. A. Kostelecký and S. Samuel, "Self-maintained coherent oscillations in dense neutrino gases," Phys. Rev. D 52, 621 (1995) [hep-ph/9506262].

[25] V. A. Kostelecký and S. Samuel, "Neutrino oscillations in the early universe with nonequilibrium neutrino distributions," Phys. Rev. D 52, 3184 (1995) [hep-ph/9507427].

[26] S. Samuel, "Bimodal coherence in dense selfinteracting neutrino gases," Phys. Rev. D 53, 5382 (1996) [hep-ph/9604341].

[27] V. A. Kostelecký and S. Samuel, "Nonequilibrium neutrino oscillations in the early universe with an inverted neutrino mass hierarchy," Phys. Lett. B 385, 159 (1996) [hep-ph/9610399].

[28] J. Pantaleone, "Stability of incoherence in an isotropic gas of oscillating neutrinos," Phys. Rev. D 58, 073002 (1998).

[29] K. N. Abazajian, J. F. Beacom and N. F. Bell, "Stringent constraints on cosmological neutrino antineutrino asymmetries from synchronized flavor transformation," Phys. Rev. D 66, 013008 (2002) [astro-ph/0203442].

[30] A. D. Dolgov, S. H. Hansen, S. Pastor, S. T. Petcov, G. G. Raffelt and D. V. Semikoz, "Cosmological bounds on neutrino degeneracy improved by flavor oscillations," Nucl. Phys. B 632 (2002) 363 [hep-ph/0201287].

[31] Y. Y. Y. Wong, "Analytical treatment of neutrino asymmetry equilibration from flavour oscillations in the early universe," Phys. Rev. D 66, 025015 (2002) [hep-ph/0203180].

[32] S. Pastor, G. G. Raffelt and D. V. Semikoz, "Physics of synchronized neutrino oscillations caused by self-interactions," Phys. Rev. D 65, 053011 (2002) [hep-ph/0109035].

[33] R. F. Sawyer, "Speed-up of neutrino transformations in a supernova environment," Phys. Rev. D 72, 045003 (2005) [hep-ph/0503013].

[34] G. G. Raffelt and G. Sigl, "Self-induced decoherence in dense neutrino gases," Phys. Rev. D 75, 083002 (2007) [hep-ph/0701182].

[35] J. T. Pantaleone, "Neutrino flavor evolution near a supernova's core," Phys. Lett. B 342, 250 (1995) [astro-ph/9405008].

[36] Y. Z. Qian and G. M. Fuller, "Neutrino-neutrino scattering and matter enhanced neutrino flavor transformation in Supernovae," Phys. Rev. D 51, 1479 (1995) [astro-ph/9406073].

[37] G. Sigl, "Neutrino mixing constraints and supernova nucleosynthesis," Phys. Rev. D 51, 4035 (1995) [astro-ph/9410094].

[38] S. Pastor and G. Raffelt, "Flavor oscillations in the supernova hot bubble region: Nonlinear effects of neutrino background," Phys. Rev. Lett. 89, 191101 (2002) [astro-ph/0207281].

[39] A. B. Balantekin and H. Yüksel, "Neutrino mixing and nucleosynthesis in core-collapse supernovae," New J. Phys. 7, 51 (2005) [astro-ph/0411159].

[40] H. Duan, G. M. Fuller, J. Carlson and Y. Z. Qian, "Coherent development of neutrino flavor in the supernova environment," Phys. Rev. Lett. 97, 241101 (2006) [astro-ph/0608050].

[41] G. M. Fuller and Y. Z. Qian, "Simultaneous flavor transformation of neutrinos and antineutrinos with dominant potentials from neutrino neutrino forward scattering," Phys. Rev. D 73, 023004 (2006) [astro-ph/0505240]. 
[42] H. Duan, G. M. Fuller and Y. Z. Qian, "Collective neutrino flavor transformation in supernovae," Phys. Rev. D 74, 123004 (2006) [astro-ph/0511275].

[43] S. Hannestad, G. G. Raffelt, G. Sigl and Y. Y. Y. Wong, "Self-induced conversion in dense neutrino gases: Pendulum in flavour space," Phys. Rev. D 74, 105010 (2006) [astro-ph/0608695].

[44] H. Duan, G. M. Fuller, J. Carlson and Y. Z. Qian, "Analysis of Collective Neutrino Flavor Transformation in Supernovae," Phys. Rev. D 75, 125005 (2007) [astro-ph/0703776].

[45] G. G. Raffelt and A. Y. Smirnov, "Self-induced spectral splits in supernova neutrino fluxes," Phys. Rev. D 76, 081301 (2007), arXiv:0705.1830 [hep-ph].

[46] H. Duan, G. M. Fuller and Y. Z. Qian, "A Simple Picture for Neutrino Flavor Transformation in Supernovae," Phys. Rev. D 76, 085013 (2007), arXiv:0706.4293 [astro-ph].

[47] H. Duan, G. M. Fuller, J. Carlson and Y. Z. Qian, "Neutrino Mass Hierarchy and Stepwise Spectral Swapping of Supernova Neutrino Flavors," arXiv:0707.0290 [astro-ph].

[48] G. G. Raffelt and A. Yu. Smirnov, "Adiabaticity and spectral splits in collective neutrino flavor transformations," arXiv:0709.4641 [hep-ph].

[49] A. Esteban-Pretel, S. Pastor, R. Tomàs, G. G. Raffelt and G. Sigl, "Decoherence in supernova neutrino transformations suppressed by deleptonization," arXiv:0706.2498 [astro-ph].

[50] Y. Z. Qian, G. M. Fuller, G. J. Mathews, R. Mayle, J. R. Wilson and S. E. Woosley, "A Connection Between Flavor Mixing Of Cosmologically Significant Neutrinos And Heavy Element Nucleosynthesis In Supernovae," Phys. Rev. Lett. 71, 1965 (1993).

[51] G. Sigl and G. Raffelt, "General kinetic description of relativistic mixed neutrinos," Nucl. Phys. B 406, 423 (1993).

[52] H. Goldstein, "Classical Mechanics" (Addison-Wesley, Reading, MA, 1980), 692 pp.

[53] L. D. Landau and E. M. Lifshitz, "Course of Theoretical Physics; Vol. I: Mechanics" (Pergamon Press, Oxford, England, 1976).

[54] G. M. Fuller, R. Mayle, B. S. Meyer and J. R. Wilson, "Can a closure mass neutrino help solve the supernova shock reheating problem?", Astrophys. J. 389, 517 (1992).

[55] A. Mirizzi and G. G. Raffelt, "New analysis of the SN 1987A neutrinos with a flexible spectral shape," Phys. Rev. D 72, 063001 (2005) [astro-ph/0508612].

[56] C. Lunardini, "The diffuse supernova neutrino flux, star formation rate and SN1987A," Astropart. Phys. 26, 190 (2006) [astro-ph/0509233].

[57] H. Yuksel and J. F. Beacom, "Neutrino spectrum from SN 1987A and from cosmic supernovae," Phys. Rev. D 76, 083007 (2007) [astro-ph/0702613].

[58] M. Malek et al. [Super-Kamiokande Collaboration], "Search for supernova relic neutrinos at SuperKamiokande," Phys. Rev. Lett. 90, 061101 (2003) [hep-ex/0209028].

[59] G. L. Fogli, E. Lisi, A. Mirizzi and D. Montanino, "Three-generation flavor transitions and decays of supernova relic neutrinos," Phys. Rev. D 70, 013001 (2004) [hep-ph/0401227].

[60] H. Duan, G. M. Fuller, J. Carlson, and Y. Z. Qian, "Flavor evolution of the neutronization neutrino burst from an O-Ne-Mg core-collapse supernova," arXiv:0710.1271 [astro-ph].

[61] A. Friedland and C. Lunardini, "Do many-particle neutrino interactions cause a novel coherent effect?," JHEP 0310, 043 (2003) [hep-ph/0307140].

[62] A. B. Balantekin and Y. Pehlivan, "Neutrino neutrino interactions and flavor mixing in dense matter," J. Phys. G 34, 47 (2007) [astro-ph/0607527].

[63] F. Iavernaro and F. Mazzia, "Solving Ordinary Differential Equations by Generalized Adams Methods: Properties and implementation techniques," Appl. Num. Math. 28, 107 (1998); "BlockBoundary Value Methods for the solution of Ordinary Differential Equation," Siam J. Sci. Comput. 21, 323 (1999). Software available at: pitagora.dm.uniba.it/ mazzia/ode/gamd.html . 